OPEN ACCESS

Edited by:

Eiman Aleem,

University of Arizona, United States

Reviewed by: Hui Li,

Northeast Agricultural University,

China

Zhuanjian Li,

Henan Agricultural University, China

*Correspondence:

Qingbin Luo

qbluo@scau.edu.cn

${ }^{\dagger}$ These authors have contributed equally to this work

Specialty section:

This article was submitted to Cell Growth and Division,

a section of the journal

Frontiers in Cell and Developmental

Biology

Received: 02 September 2020 Accepted: 04 December 2020

Published: 21 December 2020

Citation:

Chao X, Guo L, Wang Q, Huang W, Liu M, Luan K, Jiang J, Lin S, Nie Q, Luo W, Zhang X and Luo Q (2020) miR-429-3p/LPIN1 Axis Promotes Chicken Abdominal Fat Deposition via PPARy Pathway. Front. Cell Dev. Biol. 8:595637. doi: 10.3389/fcell.2020.595637

\section{miR-429-3p/LPIN1 Axis Promotes Chicken Abdominal Fat Deposition via PPAR $\gamma$ Pathway}

\author{
Xiaohuan Chao 1,2,3t, Lijin Guo 1,2,3t, Qi Wang 1,2,3, Weiling Huang 1,2,3, Manqing Liu'1,2,3, \\ Kang Luan ${ }^{1,2,3}$, Jinqi Jiang ${ }^{1,2,3}$, Shudai Lin ${ }^{1,2,3}$, Qinghua Nie ${ }^{1,2,3}$, Wen Luo ${ }^{1,2,3}$, \\ Xiquan Zhang ${ }^{1,2,3}$ and Qingbin Luo ${ }^{1,2,3 *}$ \\ ' Guangdong Laboratory for Lingnan Modern Agricultural Science and Technology, South China Agricultural University, \\ Guangzhou, China, ${ }^{2}$ Guangdong Provincial Key Lab of Agro-Animal Genomics and Molecular Breeding, and Key Laboratory \\ of Chicken Genetics, Breeding and Reproduction, Ministry of Agriculture, Guangzhou, China, ${ }^{3}$ College of Animal Science, \\ South China Agricultural University, Guangzhou, China
}

To explore the regulatory mechanism of abdominal fat deposition in broilers, 100-day-old Sanhuang chickens $(n=12)$ were divided into high-fat and low-fat groups, according to the abdominal fat ratio size. Total RNA isolated from the 12 abdominal fat tissues was used for miRNA and mRNA sequencing. Results of miRNA and mRNA sequencing revealed that miR-429-3p was highly expressed in high-fat chicken whereas LPIN1 expression was downregulated. Further, we determined that miR-429-3p promoted preadipocyte proliferation and differentiation, whereas LPIN1 exerted an opposite effect. Notably, we found that the miR-429-3p/LPIN1 axis facilitated PPAR $\gamma$ pathway activation, which is closely associated with the progression of adipogenesis. In conclusion, our results provide evidence that a novel miR-429-3p/LPIN1 axis is involved in the regulation of adipogenesis, which may have a guiding role in the improvement of breeding for abdominal fat traits in broiler chickens.

\footnotetext{
Keywords: RNA-Seq, miR-429-3p/LPIN1 axis, preadipocyte proliferation, preadipocyte differentiation, PPAR $\gamma$ pathway abstract
}

\section{INTRODUCTION}

Obesity is becoming a world-wide health problem, which is believed to increase the risk of psychological problems, fatty liver disease, cardiovascular disease, and even cancer (Bray, 2004; Swinburn et al., 2011; Hruby and Hu, 2015; Lauby-Secretan et al., 2016; Bugianesi, 2020). The rising prevalence of this disorder is arousing the concern and awareness of the public, as well as piquing the attention and interest of researchers (James, 2018; Jaacks et al., 2019; Saxton et al., 2019). The wide availability of affordable and high-fat foods and beverages are contributing to excess energy intake and increased body weight (Liu et al., 2016; Asghari et al., 2017; Heianza and Qi, 2017) with sedentary lifestyles contributing to the weight issue (Barnett et al., 2018; Lavie et al., 2019). In addition to an unhealthy lifestyle, obesity is believed to be highly associated with genetic manipulation (Goodarzi, 2018; Qasim et al., 2018). In vertebrates, adipogenesis is a complex process involving the regulation of multiple gene expression networks and various metabolic 
pathways (Homan et al., 2011; Bugianesi, 2020). A number of obesity-associated genes have been identified in adipogenesis regulation, such as melanocortin-4 receptor (MC4R) (Kuhnen et al., 2019), uncoupling protein-1 (UCP1) (Fedorenko et al., 2012), and pro-opiomelanocortin (POMC) (Qiu et al., 2014). Additionally, peroxisome proliferator-activated receptor gamma $(\operatorname{PPAR} \gamma)$ (Brun et al., 1996) and CCAAT/enhancer-binding protein alpha (CEBP $\alpha)$ (Lefterova et al., 2008) have been shown to possess the ability to mediate the terminal differentiation of adipose tissue/cell development. In the poultry industry, excessive deposition of fat in commercial broilers can lead to low economic efficiency since the public tend to favor low-fat content chicken (Fouad and El-Senousey, 2014). A diet of highfat chicken would encourage excess weight gain, posing a risk to people's health. Therefore, investigation into obesity utilizing a chicken model can gain new insights into the mechanism underlying fat deposition and provide a possible solution for the public obesity problem.

In the past decade, the ability of microRNAs (miRNAs) and small non-coding RNAs to exert their biological functions by binding to 3'-untranslated regions (3'-UTRs) of target mRNAs, causing translational repression or degradation has become widely accepted (Bartel, 2004). Despite a lack of proteincoding ability, miRNAs are reported to play irreplaceable roles in various pathological and physiological processes including adipogenesis, metabolism and tumorigenesis (Krol et al., 2010; Li et al., 2012; Mohr and Mott, 2015; Subramaniam et al., 2019). Using miRNA sequencing analyses, we determined miR-429-3p to be a miRNA of interest for exploring the regulation of adipogenesis in chicken. miR-429 is a member of the highly conserved miR-200 family (Hasuwa et al., 2013). To date, most of the research on miR-429-3p has focused on cancer development, whereas little is known about its role in adipogenesis. The present study is the first to shed light on the effect of miR-429-3p in the mediation of adipogenesis.

LPIN1 encodes lipin-1 protein, which is reported to be responsible for lipidic and metabolic homeostasis (Phan et al., 2004). We observed a downregulation in LPIN1 in the abdominal fat tissue from high-fat chicken. In addition, LPIN1 is predicted to be a potential target of miR-429-3p in the regulation of adipogenesis in chicken.

It is well-established that PPAR $\gamma$ is a predominant regulator of adipocyte differentiation (Tontonoz et al., 1994). In the process of adipogenesis, PPAR $\gamma$ works in synergy with $\mathrm{CEBP} \alpha$ (which is also a well- known key regulator of adipocyte differentiation) to activate many other relevant molecular factors and contribute to lipid accumulation (Madsen et al., 2014). Abnormal expression of PPAR $\gamma$ in humans and insufficiency of PPAR $\gamma$ in mice can result in lipodystrophy (Campeau et al., 2012), indicating sufficient PPAR $\gamma$ expression is indispensable to the maintenance of adipogenesis.

To our best knowledge, there is no published study elucidating how the miR-429-3p/LPIN1 axis interacts with the PPAR $\gamma$ pathway in the adipogenesis of chicken. Based on sequencing data, we aimed to identify the potential role of miR-429-3p and LPIN1 in adipogenesis in a chicken model, which may better our understanding of the genetic regulatory networks in adipogenesis.

\section{MATERIALS AND METHODS}

\section{Animal Experiment and Ethics Statement}

The animals used in this study were supported by Wens. Twelve Sanhuang broilers were put in a cage after birth and were fed ad libitum. When they reach the age of 100 days, they were euthanized by cervical dislocation. Body weight and abdominal fat weight were weighed. The abdominal fat tissues were collected and saved in a $-80^{\circ} \mathrm{C}$ refrigerator. The body weight and abdominal fat weight of 12 individuals were listed in Table 1. The animal experiments conducted in this study were approved (license ID: SCAU\#2017015; September 13, 2017) by the Animal Care Committee of South China Agricultural University.

\section{Whole Genome miRNA and mRNA Screen}

Twelve 100-day-old ad libitum Sanhuang broilers were euthanized and abdominal fat tissue was collected. The animals were divided into the high-fat group and the low-fat group based on the abdominal fat ratio. Total RNA was extracted from the 12 abdominal fat tissues, were sent to BGI Technology for miRNA and mRNA sequencing.

\section{Cell Culture and Cell Transfection}

Immortalized chicken precursor adipocytes (ICP-1) was provided by Hui Li research group from Northeast Agricultural University (Heilongjiang, China). ICP-1 was cultured in DMEM/F12 medium (Gibco, CA, United States) with 15\% fetal serum (Gibco, CA, United States) and 1\% streptomycin/penicillin (Invitrogen, $\mathrm{CA}$, United States) at $37^{\circ} \mathrm{C}$ with $5 \% \mathrm{CO}_{2}$. The DMEM (Gibco, CA, United States) with 10\% fetal serum and $1 \%$ streptomycin/penicillin was used for culturing dermal fibroblast (DF-1) cells. The culture conditions for DF-1 were the same as for ICP-1. Lipofectamine 3000 kit (Invitrogen, CA, United States)

TABLE 1 | Abdominal fat rate of 12 Sanhuang broilers.

\begin{tabular}{lccc}
\hline Individual ID & Weight (g) & $\begin{array}{c}\text { Abdominal fat } \\
\text { weight } \mathbf{( g )}\end{array}$ & $\begin{array}{c}\text { Abdominal } \\
\text { fat rate (\%) }\end{array}$ \\
\hline L1 & 1705.9 & 66.4 & 3.89 \\
L2 & 1550.0 & 56.8 & 3.66 \\
L3 & 1360.0 & 50.3 & 3.70 \\
L4 & 1564.8 & 58.2 & 3.72 \\
L5 & 1419.9 & 55.6 & 3.92 \\
L6 & 1235.2 & 34.7 & 2.81 \\
H1 & 1602.5 & 128.8 & 8.04 \\
H2 & 1747.2 & 146.5 & 8.38 \\
H3 & 1415.8 & 119.9 & 8.47 \\
H4 & 1735.5 & 151.6 & 8.74 \\
H5 & 1618.9 & 155.5 & 9.61 \\
H6 & 1839.7 & 179.4 & 9.75 \\
\hline
\end{tabular}


was used for cell transfection of plasmids and oligonucleotides described in next section as per the manufacturer's protocol. Transfection dose of the plasmids or oligonucleotides for different plates was as follows: $2.5 \mu \mathrm{g} /$ well or $125 \mathrm{nM} /$ well for 6-well plates, $1 \mu \mathrm{g} /$ well or $50 \mathrm{nM}$ /well for 12-well plates, and $0.25 \mu \mathrm{g} /$ well or $12.5 \mathrm{nM}$ /well for 48 -well plates.

\section{Plasmids Construction and Oligonucleotides Synthesis}

The complete CDS sequence of LPIN1 (XM_004935773.3) was synthesized by GeneCreate (Hubei, China) and subcloned into $X b a \mathrm{I}$ and XhoI site in pcDNA3.1 vector (Promega, WI, United States). The plasmid was named as pcDNA3.1- LPIN1. Further, gga-miR-429-3p mimic, gga-miR-429-3p inhibitor, and siRNA of LPIN1 were synthesized by RioBio (Guangdong, China). Oligonucleotides sequences used are listed in Table 2.

\section{Total RNA Extraction}

MagZol Reagent (Magen, Guangdong, China) was used for extracting total RNA from abdominal fat tissues and cells according to manufacturer's instructions. A total of $50 \mathrm{mg}$ homogenized tissues or $10^{6}$ cells were lysed by adding $1 \mathrm{~mL}$ MagZol Reagent at $25^{\circ} \mathrm{C}$. Next, $200 \mu \mathrm{L}$ chloroform (Damao, Tianjin, China) was added to the mixture and centrifuged at $12,000 \times g$ at $4^{\circ} \mathrm{C}$ for $15 \mathrm{~min}$. The supernatant was transferred to a new $1.5 \mathrm{~mL}$ tube, to which $500 \mu \mathrm{L}$ isopropyl alcohol (Damao, Tianjin, China) was added, mixed well, and incubated at room temperature for $10 \mathrm{~min}$. The mixture was centrifuged at $12,000 \times g$ at $4^{\circ} \mathrm{C}$ for $10 \mathrm{~min}$ and the supernatant was discarded. Then, $1 \mathrm{~mL}$ of $75 \%$ alcohol (Damao, Tianjin, China) was used to wash the pellet followed by centrifugation. Finally, $40 \mu \mathrm{L}$ RNasefree water (TsingKe, Beijing, China) was used to dissolve the RNA pellet and stored at $-80^{\circ} \mathrm{C}$ until further analysis.

\section{Reverse Transcription and Real-Time Quantitative PCR (qPCR)}

HiScript ${ }^{\circledR}$ II Q RT SuperMix for qPCR (+ gDNA wiper) (Vazyme, Jiangsu, China) and miRNA 1st Strand cDNA Synthesis Kit (by stem-loop) (Vazyme, Nanjing, China) were used for reverse transcription PCR for mRNA and miRNA, respectively, following manufacturer's protocol. Quantitative PCR (qPCR) was performed using ABI QuantStudio 5 instrument (Thermo Fisher, NY, United States) by using ChamQ Universal SYBR qPCR Master Mix (Vazyme, Nanjing, China). GAPDH and U6 were used as internal controls for mRNA and miRNA qPCR, respectively. The primers used in qPCR were designed by Premier Primer 5.0 software and the primer sequences are listed in Supplementary Table 1.

TABLE 2 | Oligonucleotides sequence information.

\begin{tabular}{ll}
\hline Fragment name & Fragment sequence $\mathbf{( \mathbf { 5 } ^ { \prime } \mathbf { - } \mathbf { 3 } ^ { \prime } \mathbf { ) }}$ \\
\hline gga-miR-429-3p mimic & UAAUACUGUCUGGUAAUGCCGU \\
gga-miR-429-3p inhibitor & ACGGCAUUACCAGACAGUAUUA \\
si-LPIN1 & GTTCCTTGATCAAAGCTAA \\
\hline
\end{tabular}

\section{Flow Cytometry Analysis}

After $48 \mathrm{~h}$ of transfection, 0.25\%-EDTA trypsin (Gibco, CA, United States) was used to digest the cells and $1 \mathrm{~mL}$ culture medium with $15 \%$ fetal serum was used to terminate the digestion reaction. The cell suspension was centrifuged at $2,000 \times g$ at $4^{\circ} \mathrm{C}$ for $5 \mathrm{~min}$. The cell pellet was washed twice with $1 \mathrm{~mL}$ ice-cold PBS (Gibco, CA, United States). Subsequently, the cells were fixed overnight with 70\% alcohol (Damao, Tianjin, China). Next, $1 \mathrm{~mL}$ ice-cold PBS (Gibco, CA, United States) was used to wash cells sediment and cells were stained with $0.5 \mathrm{~mL}$ Propidium iodide (PI) solution (Beyotime, Shanghai, China) at $37^{\circ} \mathrm{C}$ in dark for $30 \mathrm{~min}$. Finally, the cells were analyzed by a CytoFlex instrument (Beckman, CA, United States).

\section{Oil Red O Staining}

The transfected cells were induced to differentiate into adipocytes by the culture medium with $15 \%$ fetal serum and $0.2 \%$ oleic acid (Sigma, CA, United States). The cells were washed with PBS and then, $4 \%$ formaldehyde was added to fix the cells for $30 \mathrm{~min}$. Next, the cells were washed with PBS, followed by wash with $60 \%$ isopropanol, and finally stained with oil red $\mathrm{O}$ reagent for $30 \mathrm{~min}$. The cells were then treated with $60 \%$ isopropanol for $15 \mathrm{~s}$ and rinsed with PBS 3 times for $3 \mathrm{~min}$ each time. The stained cells were observed, and images were captured under an electron microscope (Nikon, Tokyo, Japan). Oil red O dye was extracted from the cells in isopropanol solution and quantified in a microreader (Bio-Rad, CA, United States) at $510 \mathrm{~nm}$. Cells were collected and resuspended in a $1.5 \mathrm{~mL}$ tube with $1 \mathrm{~mL}$ PBS. Finally, cells were counted in a cell counter (CountStar, Shanghai, China).

\section{5-Ethynyl-2' -Deoxyuridine (EdU) Assay}

Cell-Light EdU Apollo567 In Vitro Kit (RioBio, Guangdong, China) was used to detect the cell proliferation activity by following manufacturer's protocol. After staining, cells were captured in at least three random visual fields using a DMi8 microscope (Leica, Wetzlar, Germany).

\section{Western Blotting}

Protein was extracted from the cells inoculated in a 6well plate that had been transfected for $48 \mathrm{~h}$ by using icecold radio immunoprecipitation (RIPA) lysis buffer (Beyotime, Shanghai, China) with $1 \mathrm{mM}$ phenylmethyl sulfonyl fluoride (Beyotime, Shanghai, China). Protein samples were separated on a $12 \%$ SDS-PAGE gel (EpiZyme, Shanghai, China) run at $120 \mathrm{~V}$ for $60 \mathrm{~min}$. Then, the proteins were transferred onto the polyvinylidene fluoride (PVDF) membrane (Bio-Rad, CA, United States). After 30 min blocking with 5\% skim milk, the membrane was incubated with anti-LPIN1 (1:1,000; 863631, ZENBIO), anti-PPAR $\gamma$ (1:1,000; bs-0530R, BIOSS), anti$\mathrm{CEBP} \alpha(1: 1,000 ; \mathrm{LS}-\mathrm{B} 4685, \mathrm{LABIO})$ or anti-GAPDH antibodies (1:2,000; bsm-33033M, BIOSS) at $4^{\circ} \mathrm{C}$ overnight. Anti-mouse IgG HRP-antibody $(1: 10,000 ; 7076 \mathrm{P} 2, \mathrm{CST})$ or anti-rabbit IgG HRP-antibody (1:10,000; 7074P2, CST) were used as secondary antibodies. DAB Horseradish Peroxidase Color Development Kit 
(Beyotime, Shanghai, China) was used for chromogenic reaction following manufacturer's protocol. Finally, protein bands were visualized using Odyssey instrument (Li-cor, CA, United States) and the gray value (Image density) of protein bands was measured by Image J software.

\section{Dual-Luciferase Reporter Assay}

The wild type LPIN1 3' UTR (LPIN1-WT) containing miR429-3p binding sites and mutant type of LPIN1 3' UTR (LPIN1-MT) with altered miR-429-3p binding sites were amplified by PCR and subcloned into pmirGLO vector (Promega, WI, United States). $4 \times 10^{4}$ ICP-1 were seeded into a 96-well plate and co-transfected with miR-429-3p mimic or mimic NC with LPIN1-WT or LPIN1-MT. Firefly and Renilla Luciferase activity was detected using Dual-GLO Luciferase Assay System kit (Promega, WI, United States) in a Fluorescence/Multi-Detection Microplate Reader (BioTek, VT, United States); firefly luciferase activity was normalized to Renilla luminescence.

\section{Data Analysis}

The data represents mean \pm SEM. Student's $t$-test and ANOVAanalysis were performed to determine the statistical significance of differences observed between groups. ${ }^{*} P<0.05$; ${ }^{* *} P<0.01$; ${ }^{* * *} P<0.001 ;{ }^{* * * *} P<0.0001$; ns: no significance.

\section{RESULTS}

\section{Genome-Wide mRNA Screen and Analysis Identifies Several Differentially Expressed Genes in High-Fat Tissues}

To explore the regulatory mechanisms underlying chicken abdominal fat deposition, mRNA sequencing was performed. The abdominal fat tissues from 6 High fat (HF) broilers and 6 Low fat (LF) broilers were collected for mRNA sequencing to perform the genome-wide mRNA screen. Using mRNA sequencing, we obtained at least $21.94 \mathrm{M}$ raw reads for each sample (Supplementary Table 2) and all raw data was submitted to the NCBI SRA database (accession number: PRJNA656618; $\operatorname{link}^{1}$ ). The clean data was mapped to chicken GRCg6a genome and gene set analysis showed that a total of 18,165 genes were detected. | Fold change | $\geq 2$ and $q$-value $\leq 0.001$ were considered as the cut-off for differentially expressed genes (DEGs), and we obtained 1,562 DEGs from mRNA sequencing. Compared with the LF group, 963 genes were upregulated in HF group whereas 599 genes were down-regulated (Figure 1A). We performed classification and enrichment analysis of KEGG biological pathways for these DEGs. A total of 55 and 42 genes were classified into the lipid metabolism and carbohydrate metabolism pathways, respectively (Figure 1B). The top 20 enriched KEGG pathways are shown in Figure 1C, including complement and coagulation cascades, graft-versus-host disease, and fat

${ }^{1}$ https://dataview.ncbi.nlm.nih.gov/object/PRJNA656618 digestion and absorption among others. Furthermore, additional pathways that might be directly or mediately related to fat deposition have been listed in Supplementary Table 3. Another pathway enrichment analysis was performed using DAVID 6.8 platform, which showed that these DEGs were enriched in steroid hormone biosynthesis, metabolic pathways, PPAR signaling, ECM-receptor interaction, and glycerolipid metabolism pathways (Supplementary Table 4). The genes at the intersection of the two kinds of pathway enrichment analysis were selected. These DEGs related to fat formation and lipid metabolism are listed in Table 3, and were considered as potential candidates for abdominal fat deposition.

In addition, ingenuity pathway analysis (IPA) was performed to investigate the gene networks for the 1,562 DEGs. In IPA, we paid more attention to the pathways involved in fat deposition, such as, lipid metabolism, insulin receptor signaling adipogenesis pathway, and fatty acid $\beta$-oxidation I (Supplementary Table 5). Three networks were involved with lipid metabolism. Here, only the two networks with the enrichment degree score above 30 are shown (Figures 1D,E). Among the DEGs, HNF4A, APOB, NR1H4, LIPC, and LDL-cholesterol acted as the node genes.

Finally, we compared candidate DEGs from the KEGG, DAVID, and IPA analysis platforms that associated with fat deposition. The results showed 7 common DEGs, including SLC27A2, ACSL6, ABHD5, LPIN1, ADORA1, HNF4A, and SQLE.

\section{Sequencing Analysis of miRNA Expression in High-Fat Broilers and Low-Fat Broilers}

To clarify the underlying mechanism of abdominal fat deposition from an epigenetic perspective, abdominal fat tissues from $6 \mathrm{HF}$ broilers and $6 \mathrm{LF}$ broilers were collected for miRNA sequencing. Small RNA sequencing raw data was submitted to the NCBI SRA database (accession number: PRJNA657369) ${ }^{2}$. In total, we detected 1,841 miRNAs, including 886 known miRNAs and 955 predicted miRNAs (Supplementary Table 6). The first nucleotide bias of known miRNAs showed that miRNA sequence length was approximately 21-23 nt, with $22 \mathrm{nt}$ being the maximum size (Supplementary Figure 1). A total of 682 differentially expressed miRNAs (DEMs) were identified between the HF and LF group, including 130 known miRNAs. Of these, 99 known miRNAs were upregulated, while 31 known miRNAs were downregulated (Figure 2). The top 20 abundant DEMs are listed in Table 4 and included miR-126-5p, miR-148a-3p, miR-148b-3p, miR-122b-3p, miR429-3p, miR-122-5p, miR-196-5p, and miR-1416-5p. Among upregulated miRNAs, miR-126-5p, miR-148a-3p, miR-148b$3 p$, and miR-429-3p were most abundant, accounting for $259,237,180,829,138,036$, and 62,352 miRNA reads from HF group, respectively. Among the downregulated miRNAs, miR122b-3p, miR-122-5p and miR-1416-5p were most abundant, with miR-122b-3p being the most abundant in LF group at 834,699 reads.

\footnotetext{
${ }^{2}$ https://dataview.ncbi.nlm.nih.gov/object/PRJNA657369
} 


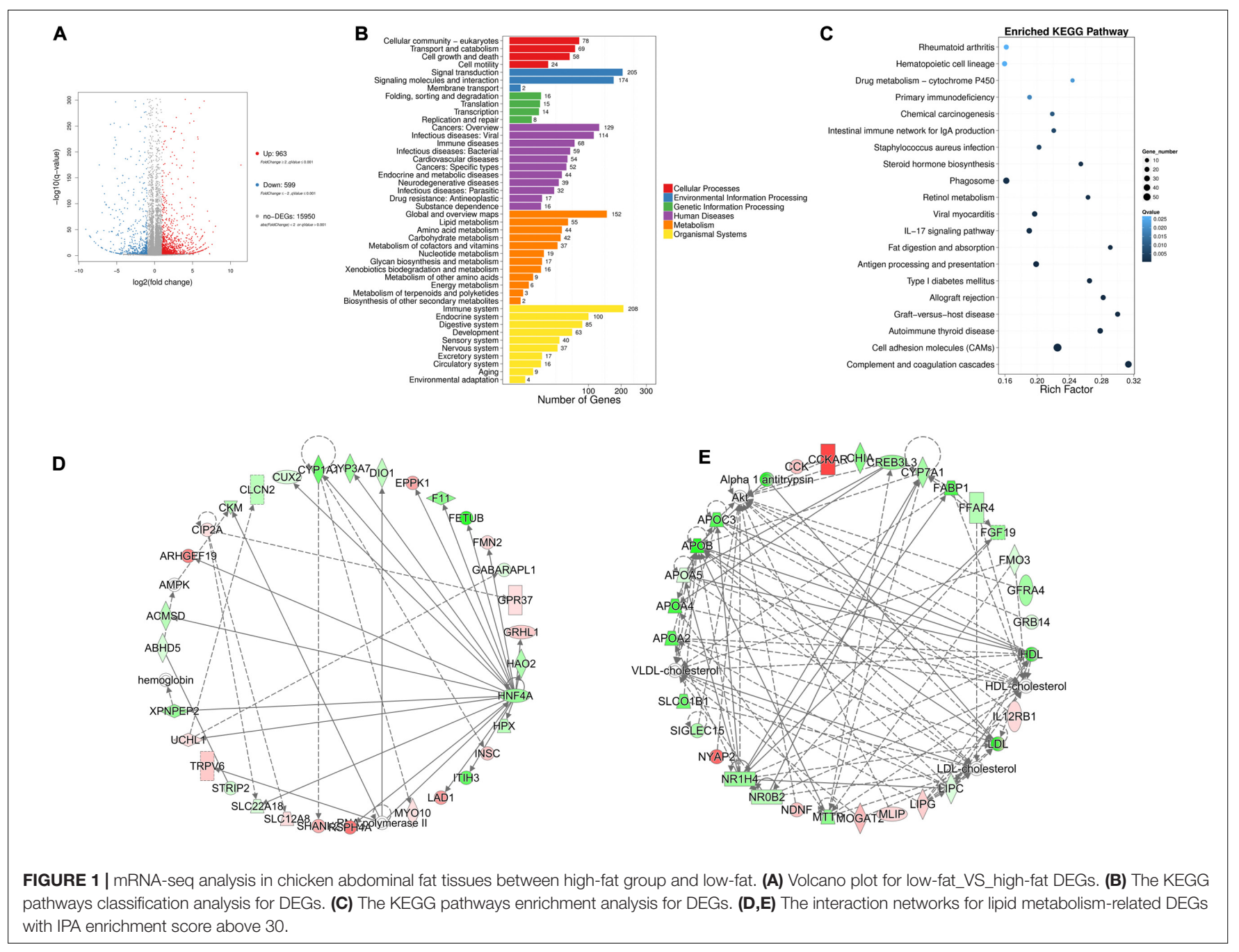

TABLE 3 | The potential candidates for abdominal fat deposition.

\begin{tabular}{lcccccl}
\hline Gene name & Low Fat Expression & High Fat Expression & log2Ratio (HF/LF) & Q value & Up Down & Gene description \\
\hline ABHD5 & 55981 & 23916 & -1.23016 & 0 & Down & adenosine A1 receptor \\
LPIN1 & 48475 & 11944 & -2.02416 & 0 & Down & lipin 1 \\
ACBD5 & 39718.81 & 19735.03 & -1.01227 & 0 & Down & acyl-CoA binding domain containing 5 \\
APOC3 & 8831 & 169 & -5.71069 & 0 & Down & apolipoprotein C3 \\
APOB & 8679 & 40 & -7.76459 & 0 & Down & apolipoprotein B \\
ADORA1 & 8141 & 3018 & -1.43482 & 0 & Down & abhydrolase domain containing 5 \\
APOA4 & 6931 & 96 & -6.17709 & 0 & Down & apolipoprotein A4 \\
FABP1 & 1328 & 21 & -5.98593 & 0 & Down & fatty acid binding protein 1 \\
DHCR24 & 1299.03 & 3065.5 & 1.235484 & $4 \mathrm{E}-160$ & Up & $24-$ dehydricholesterol reductase \\
HSD11B1b & 837 & 125 & -2.7465 & $1.5 \mathrm{E}-128$ & Down & hydroxysteroid (11-beta) dehydrogenase 1b \\
APOA5 & 820 & 338 & -1.2818 & $1.56 \mathrm{E}-46$ & Down & apolipoprotein A5 \\
SQLE & 713 & 1538 & 1.105878 & $3.64 \mathrm{E}-68$ & Up & squalene epoxidase \\
SLC27A2 & 708 & 320 & -1.14888 & $3.17 \mathrm{E}-34$ & Down & solute carrier family 27 \\
ACSL6 & 193.08 & 405.77 & 1.06826 & $3.51 \mathrm{E}-18$ & Up & long-chain-fatty acid CoA ligase 6 \\
HNF4A & 96.42 & 8.98 & -3.42775 & $1.51 \mathrm{E}-19$ & Down & Hepatocyte nuclear factor 4 alpha \\
ELOVL2 & 55 & 1 & -5.78456 & $1.74 \mathrm{E}-14$ & Down & ELOVL fatty acid elongase 2 \\
CYP7A1 & 22 & 3 & -2.87767 & $4.5 \mathrm{E}-05$ & Down & cytochrome P450 family 7 subfamily A member 1 \\
\hline
\end{tabular}



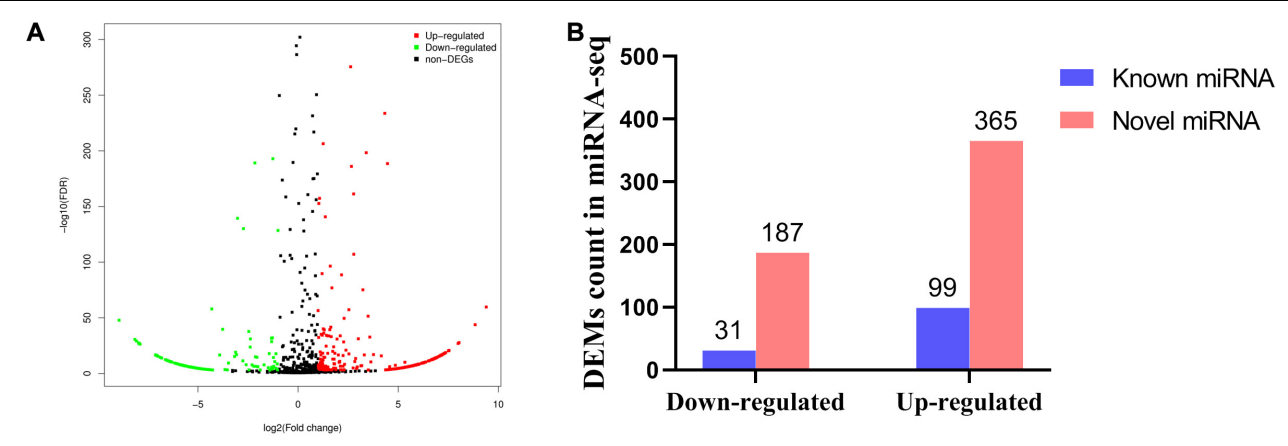

FIGURE 2 | Differentially expressed miRNA in abdominal fat between low fat broilers and high fat broilers. (A) Volcano plot for low-fat_VS_high-fat DEMs. (B) The known and novel miRNAs (down-regulated or up-regulated) from DEMs.

TABLE 4 | The top 20 abundant DEMs in HF_VS_LF.

\begin{tabular}{|c|c|c|c|c|c|}
\hline miRNA id & Read count (Low fat) & Read count (High fat) & log2Ratio (High fat/Low fat) & Up down regulation & $Q$ value \\
\hline gga-miR-122b-3p & 834699 & 105526 & -2.93401 & DOWN & 0 \\
\hline gga-miR-126-5p & 132045 & 259237 & 1.022885 & UP & 0 \\
\hline gga-miR-122-5p & 318046 & 43784 & -2.81112 & DOWN & 0 \\
\hline gga-miR-148a-3p & 73733 & 180829 & 1.343887 & UP & 0 \\
\hline gga-miR-148b-3p & 44329 & 138036 & 1.688365 & UP & 0 \\
\hline gga-miR-1416-5p & 93406 & 30166 & -1.58095 & DOWN & 0 \\
\hline gga-miR-429-3p & 20400 & 62352 & 1.66151 & UP & 0 \\
\hline gga-miR-196-5p & 9988 & 34948 & 1.856585 & UP & 0 \\
\hline gga-miR-200a-3p & 2842 & 27316 & 3.314411 & UP & 0 \\
\hline gga-miR-200b-3p & 4731 & 21781 & 2.252496 & UP & 0 \\
\hline gga-miR-375 & 13756 & 3176 & -2.06514 & DOWN & 0 \\
\hline gga-miR-130c-3p & 4342 & 8549 & 1.027039 & UP & 0 \\
\hline gga-miR-135a-5p & 2506 & 9942 & 2.037793 & UP & 0 \\
\hline gga-let-7l-5p & 224 & 11707 & 5.757372 & UP & 0 \\
\hline gga-miR-15b-5p & 3518 & 8147 & 1.261156 & UP & 0 \\
\hline gga-miR-34b-3p & 1988 & 9386 & 2.288836 & UP & 0 \\
\hline gga-miR-183 & 7954 & 3256 & -1.23894 & DOWN & 0 \\
\hline gga-miR-449a & 841 & 6271 & 2.948161 & UP & 0 \\
\hline gga-miR-142-5p & 1661 & 4978 & 1.633157 & UP & 0 \\
\hline gga-miR-122-3p & 4703 & 1044 & -2.12182 & DOWN & 0 \\
\hline
\end{tabular}

\section{The Co-analysis Between DEMs and DEGs Revealed miR-429-3p/LPIN1 Axis May Regulate Adipogenesis}

To further analyze the interactions between specific miRNAs and their target genes during abdominal fat deposition, we performed a DEMs-DEGs co-analysis. Considering the negative regulation of target mRNAs by miRNAs, the target prediction was conducted between upregulated DEMs and downregulated DEGs, or downregulated DEMs and upregulated DEGs. We obtained 1,113 target pairs, among which, 62 upregulated DEMs could target 229 DEGs that formed 801 negatively correlated target pairs. Similarly, 312 target pairs were formed by 25 downregulated DEMs and 222 DEGs. Based on the target relationship between DEMs and DEGs, we constructed two miRNA-gene interaction networks. As shown in Figure 3A, there are 16 up-regulated miRNAs and 118 DEGs in this network. miR-12229-3p and miR-12290-5p target multiple DEGs and may play a significant role. Further, Figure 3B shows that 14 downregulated miRNAs can interact with 176 DEGs. Thus, it can be concluded that miR-12207-3p and miR-1699 are critical nodes and target multiple common DEGs. Considering the candidate genes screened in mRNA sequencing, we inferred that miR-429-3p and miR-107-3p can target LPIN1 and ADORA1, respectively, which are related to lipid metabolism. Compared with miR-429-3p/LPIN1 axis, both miR-107-3p and ADORA1 have lower abundance. Therefore, miR-429-3p/LPIN1 axis may be an important regulator of adipogenesis, and we focused on this axis in follow-up experiments.

\section{miR-429-3p Facilitates Preadipocyte Proliferation}

The differential expression of miR-429-3p in abdominal fat tissues of high-fat and low-fat animals was experimentally verified by qPCR (Figure 4A). A miR-429-3p mimic and 
A
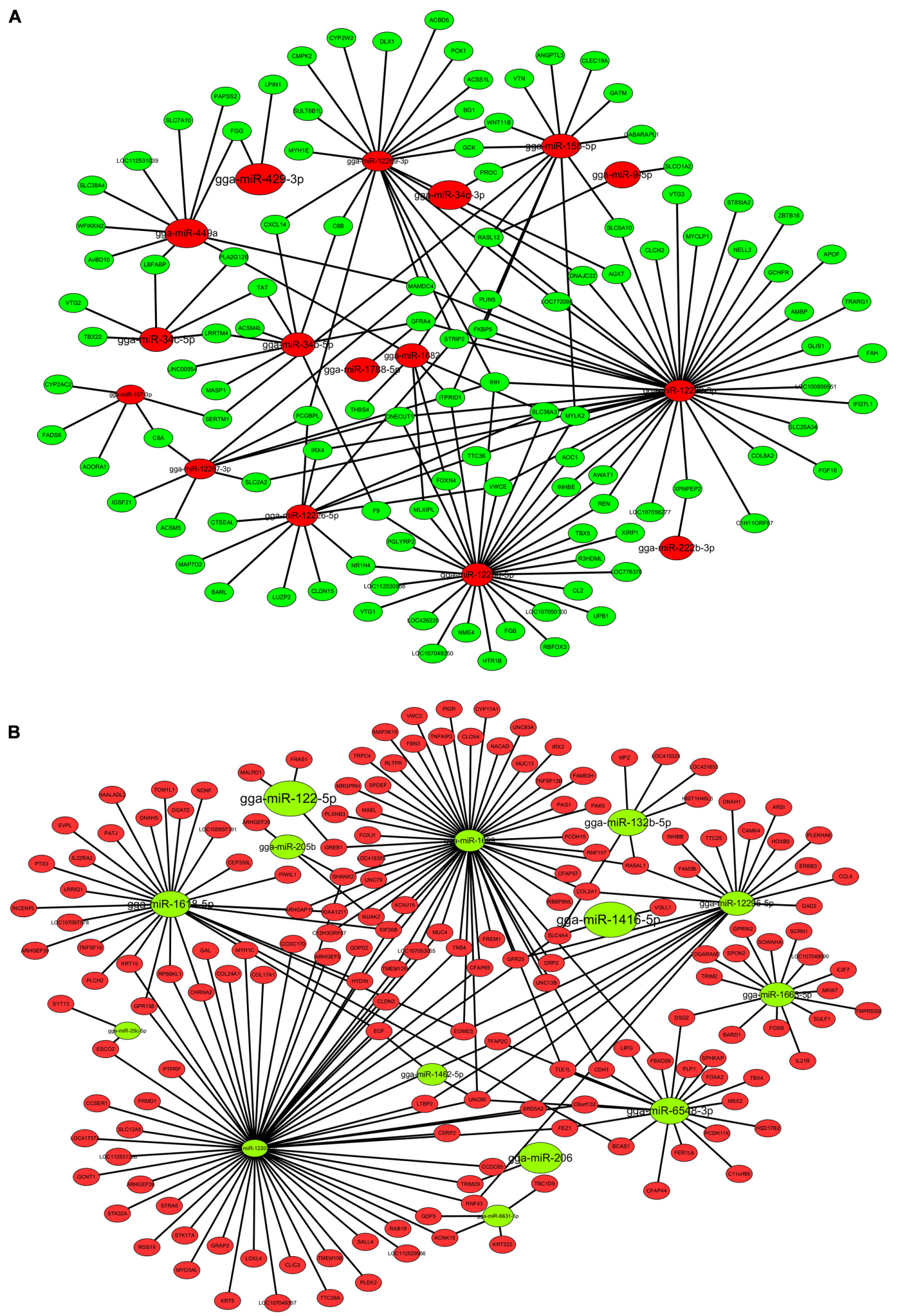

FIGURE 3 | DEMs-DEGs interaction networks. (A) The interaction network between up-regulated DEMs and down-regulated DEGs. (B) The interaction network between down-regulated DEMs and up-regulated DEGs. The pattern size of DEMs was determined by miRNA abundance the pattern size of DEGs is uniform. 

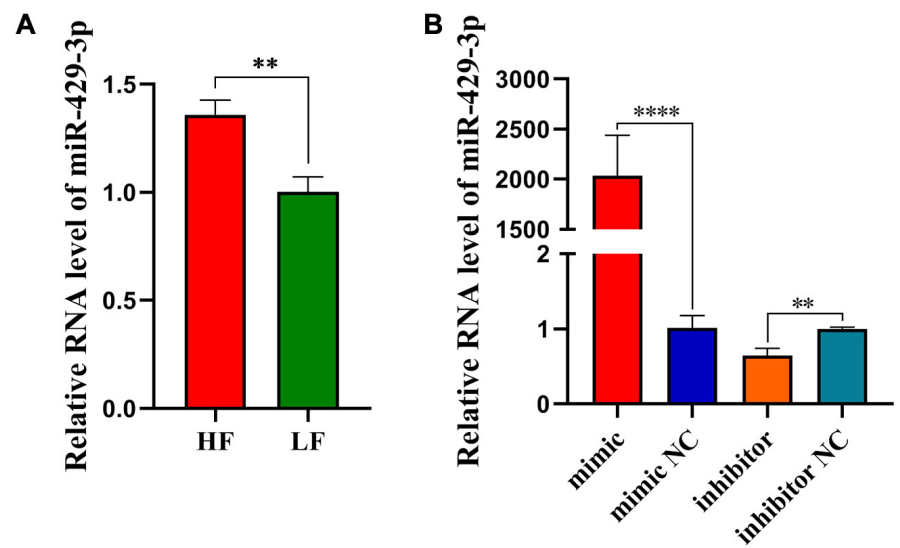

C

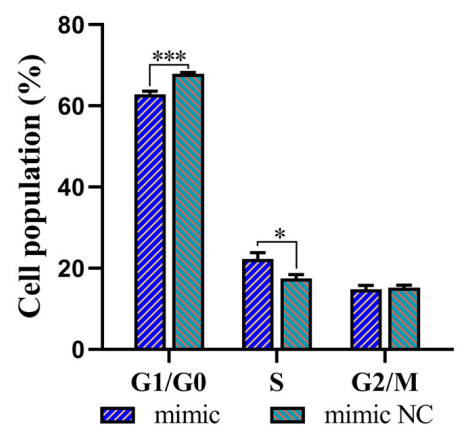

D

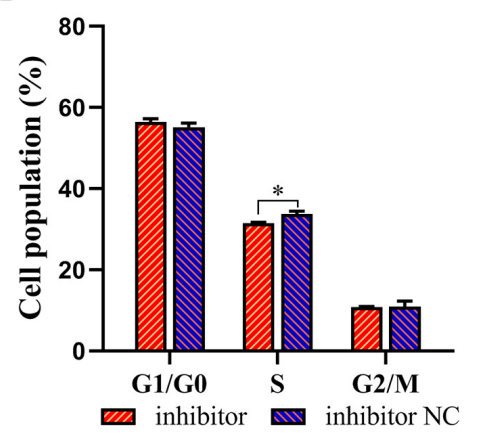

E

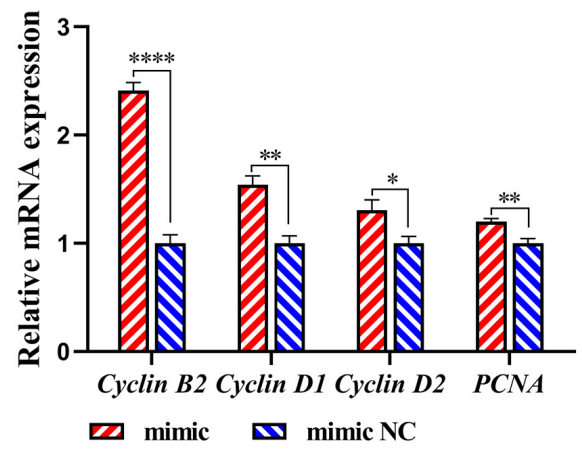

$\mathbf{F}$

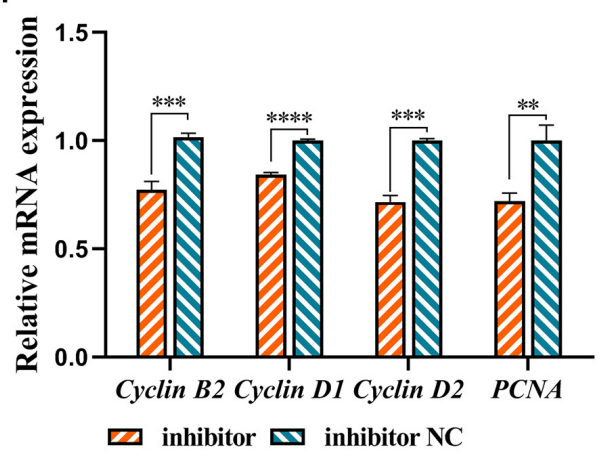

$\mathbf{G}$
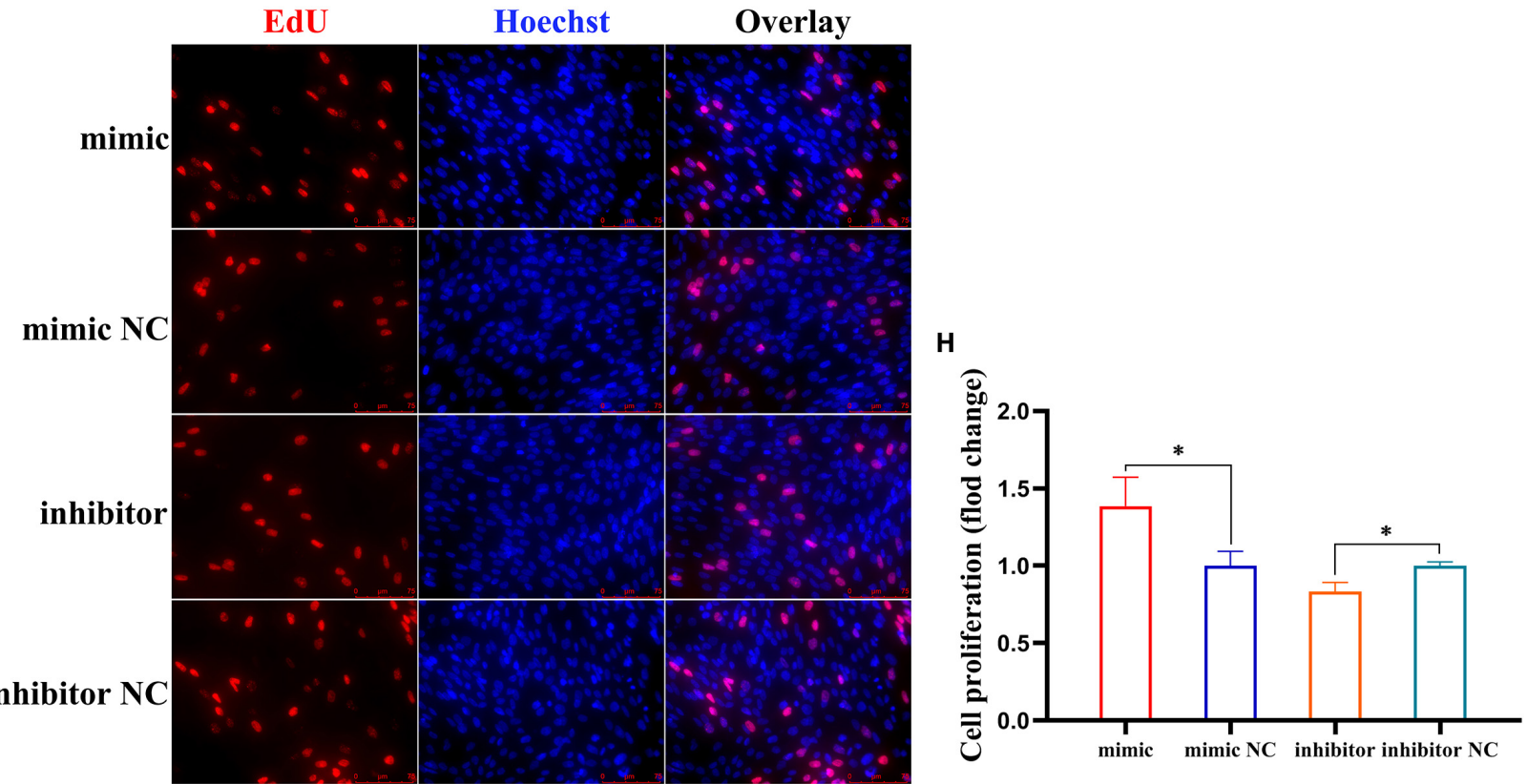

FIGURE 4 | miR-429-3p promotes preadipocytes proliferation. (A) Relative expression of miR-429-3p in high-fat chicken and low-fat chicken. (B) Transfection efficiency of miR-429-3p mimic and inhibitor. (C,D) Flow cytometry for cell cycle detection of ICP-1 cells. (E,F) Relative mRNA expression of Cyclin B2, Cyclin D1, Cyclin D2, and PCNA in ICP-1 cells. (G,H) EdU staining for cell proliferation of ICP-1 cells. Data presented as mean \pm SEM, ${ }^{*} P<0.05 ;{ }^{* \star} P<0.01 ;{ }^{\star \star \star} P<0.001$; ${ }^{\star \star \star \star} P<0.0001$; ns: no significance. 
miR-429-3p inhibitor were synthesized to explore the role of miR-429-3p in preadipocyte proliferation and differentiation. Our results showed that miR-429-3p mimic increased the expression of miR-429-3p in ICP-1 cells by as much as 2,000 folds compared with the control group. The miR-429$3 \mathrm{p}$ inhibitor could significantly knock down the endogenous expression of miR-429-3p, with an efficiency of more than $30 \%$ (Figure 4B). Flow cytometry analysis showed that miR429-3p overexpression promoted cell cycle transition from G1 to $S$ phase, while miR-429-3p knockdown arrested the cell cycle in the G1 phase, resulting in delayed cell cycle progression (Figures 4C,D). In addition, we also found that miR429-3p overexpression upregulated transcription of cell cyclepromoting genes, including cyclin B2, cyclin D1, cyclin D2, and PCNA (Figures 4E,F). EdU assay performed to observe the role of miR-429-3p in preadipocyte proliferation confirmed the positive role of $\mathrm{miR}-429-3 \mathrm{p}$ in preadipocyte proliferation (Figures 4G,H). Thus, our results provide evidence that miR429-3p can promote preadipocyte proliferation, a major process in abdominal fat deposition.

\section{miR-429-3p Contributes to Preadipocyte Differentiation via PPAR $\gamma$ Pathway}

The differentiation of preadipocytes to adipocytes is a critical process in abdominal fat deposition. In our study, miR429-3p expression was detected before and after inducing preadipocyte differentiation. We observed that miR-429$3 \mathrm{p}$ was upregulated after preadipocyte differentiation (Figure 5A). The upregulation of miR-429-3p in preadipocyte differentiation and in the abdominal fat tissue of highfat animals indicated that miR-429-3p was likely to play a positive role in abdominal fat deposition. We further verified whether miR-429-3p had an effect on the lipid droplet formation during preadipocyte differentiation. Compared with the control group, miR-429-3p overexpression enhanced lipid droplet formation during preadipocyte differentiation (Figures 5B,D); in contrast, the knockdown of miR-429-3p suppressed the formation of lipid droplets (Figures $5 \mathbf{C , E}$ ). Collectively, these results demonstrate a positive effect of miR-429-3p on promoting lipid droplet formation during the adipocyte differentiation.

Next, we investigated expression of the preadipocyte differentiation-related $C / E B P \alpha$ and $C / E B P \beta$ by qPCR. Considering that PPAR $\gamma$ pathway is widely regarded as a key process affecting adipocyte differentiation, we also verified the expression of genes involved in adipocyte differentiation in PPAR $\gamma$ pathway. After miR-429-3p mimic transfection, the mRNA levels of $C / E B P \alpha$ and $C / E B P \beta$ were significantly upregulated (Figure 5F). On the contrary, the knockdown of miR-429-3p reduced their expression (Figure 5G). Additionally, as shown in Figure $\mathbf{5 H}$, the $\mathrm{C} / \mathrm{EBP} \alpha$ and $\operatorname{PPAR} \gamma$ protein levels rose substantially after miR-429-3p overexpression, while miR429-3p knockdown reduced the protein levels of $\mathrm{C} / \mathrm{EBP} \alpha$ and PPAR $\gamma$. The change in protein levels of $\mathrm{C} / \mathrm{EBP} \alpha$ and $\operatorname{PPAR} \gamma$ modulated by miR-429-3p indicates that miR-429-3p promotes preadipocyte differentiation by activating $\operatorname{PPAR} \gamma$ pathway.

\section{LPIN1 Is a Target Gene of miR-429-3p}

LPIN1 was predicted as a target of miR-429-3p (Figure $3 \mathbf{A}$ ). The prediction result showed that miR-429-3p can target and bind to the position 3271-3277 of LPIN1 3' UTR (Figure 6A). We also performed the target pair stability analysis and the results showed that the binding between miR-429-3p and LPIN1 3' UTR is stable (Figure 6B). At the cellular level, we performed a dualluciferase reporter system analysis to verify the target relationship between miR-429-3p and LPIN1 3' UTR. Compared with other groups, the co-transfection of miR-429-3p mimic and LPIN1WT plasmid led to a lower fluorescence intensity, indicating that miR-429-3p was indeed capable of binding to LPIN1 3' UTR (Figure 6C). Further, qPCR and western blot were performed to verify whether miR-429-3p overexpression or knockdown affected LPIN1 expression at mRNA and protein levels. As shown in Figure 6D, LPIN1 expression was downregulated when miR-429-3p was overexpressed; in contrast, the expression was upregulated when miR-429-3p was knocked down. Moreover, miR-429-3p hindered the translation of LPIN1 mRNA, leading to a decrease in the protein level of LPIN1 (Figures 6E,F). Results of RNA-seq showed that LPIN1 was downregulated in abdominal fat tissues of high-fat animals. We also quantified the relative mRNA expression in abdominal fat tissues from the two groups by qPCR and observed lower expression of LPIN1 in high-fat individuals (Figure 6G). Our results confirmed that LPIN1 is a target gene of miR-429-3p and that its expression is modulated by miR-429-3p.

In order to further demonstrate whether miR-429-3p achieve its regulation of the biological function of preadipocytes by targeting LPIN1, we carried out rescue verification. We constructed the plasmid and siRNA to overexpress or knock down LPIN1. Results of qPCR and western blot showed that the transfections of pcDNA3.1-LPIN1 and si-LPIN1 led to overexpression and inhibition of LPIN1 mRNA and protein expression, respectively (Figures 7A,B). qPCR results showed that LPIN1 is able to fall down or reverse the promotion of miR-429-3p on regulating cell cycle-promoting genes (Cyclin B2, Cyclin D1, Cyclin D2, and PCNA) (Figure 7C). In addition, EdU assay also indicated that the expression of LPIN1 can restore the promotion of miR-429-3p on preadipocytes proliferation (Figures 7D,E). For preadipocytes differentiation, LPIN1 has also been shown to restore or reverse the function of miR429-3p on upregulating preadipocytes diff erentiation-related genes ( $P P A R \gamma, C / E B P \alpha, C / E B P \beta, L P L$, and $A D I P O)$ (Figure $7 \mathbf{F}$ ). What's more, LPIN1 overexpression reversed the PPAR $\gamma$ and $\mathrm{C} / \mathrm{EBP} \alpha$ protein levels upregulated by miR-429-3p (Figure 7G). These results suggested a pivotal role of LPIN1 on preadipocytes proliferation and differentiation regulated by miR-429-3p.

\section{LPIN1 Suppresses Preadipocyte Proliferation}

Following LPIN1 overexpression, preadipocyte cell cycle was arrested in the G1 phase, leading to a decline of the cell proportion in the $S$ phase (Figure 8A). Conversely, LPIN1 knockdown facilitated the preadipocyte cell cycle progression (Figure 8B). LPIN1 overexpression upregulated the expression of Cyclin B2, Cyclin D1, Cyclin D2, and PCNA 
A

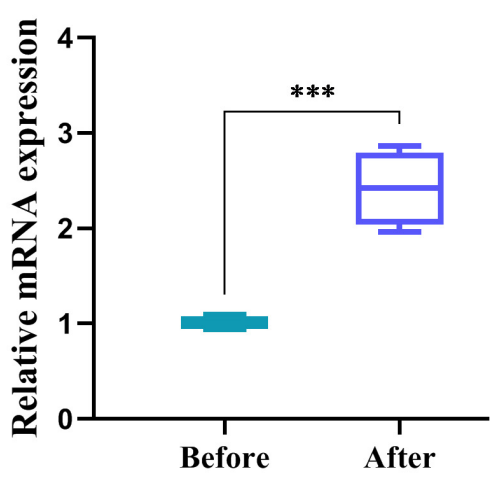

B

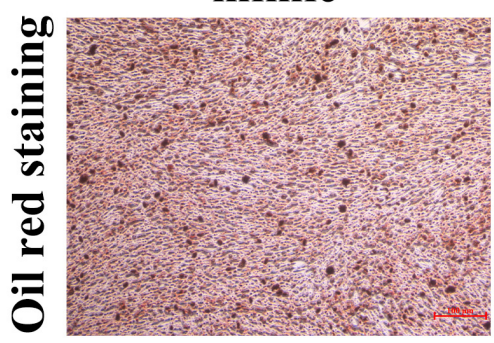

C

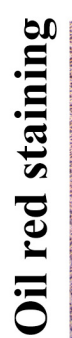

mimic NC

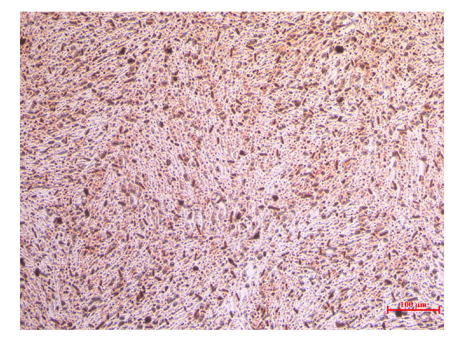

inhibitor NC

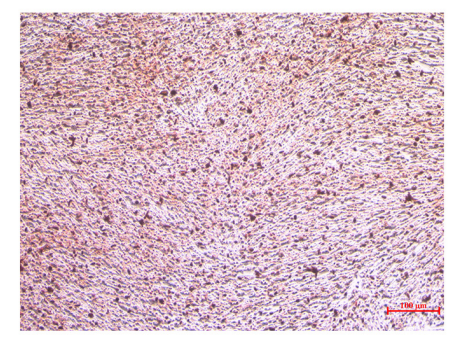

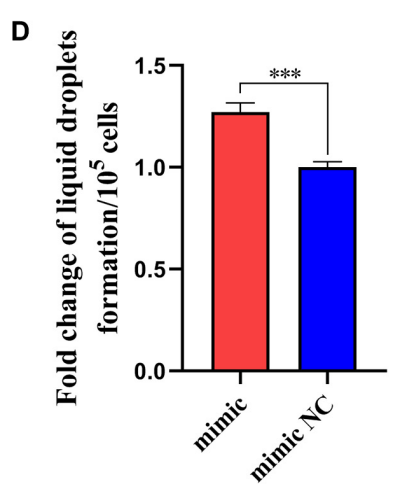

$\mathrm{E}$

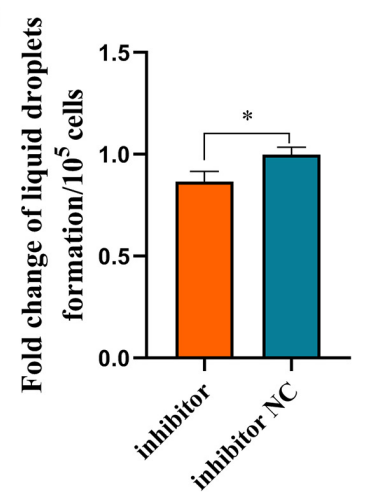

$\mathbf{F}$

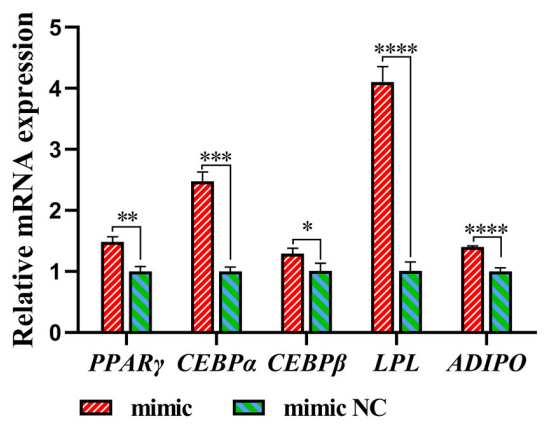

H

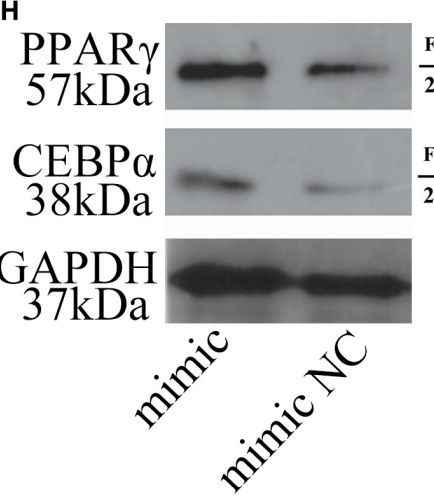

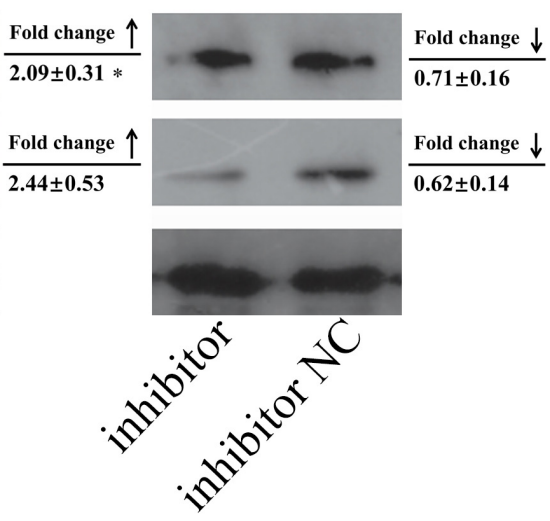

G

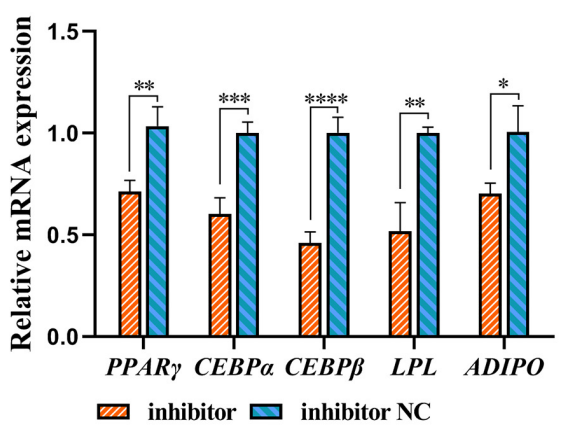

FIGURE 5 | miR-429-3p promotes preadipocytes differentiation via PPAR $\gamma$ pathway. (A) Relative expression of miR-429-3p before and after preadipocytes differentiation. (B,C) Oil red staining for lipid droplet formation. (D,E) The fold change of lipid droplets formation per $10^{5}$ cells. (F,G) Relative mRNA expression of $P P A R \gamma, C / E B P \alpha, C / E B P \beta, L P L$, and ADIPO in in ICP-1 cells. (H) The western blot for PPAR $\gamma$ and C/EBP $\alpha$ proteins in ICP-1 cells. Data presented as mean \pm SEM, ${ }^{\star} P<0.05 ;{ }^{\star \star} P<0.01 ;{ }^{* \star \star} P<0.001 ;{ }^{* \star \star *} P<0.0001 ;$ ns: no significance. 

A
gga-miR-429-3p
LPIN1 3'UTR-WT
(Position 3271-3277)
(Position 3271-3277)
LPIN1 3'UTR-MT

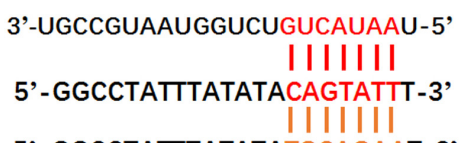

3'-UgccguaAuggucugucauaAu-5' | | | | | | |

5'-GgCCTATTTATATACAGTATTT-3' | | | | | | |

B

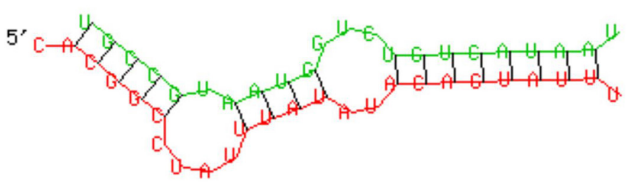

mfe: $-22.3 \mathrm{kcal} / \mathrm{mol}$

C

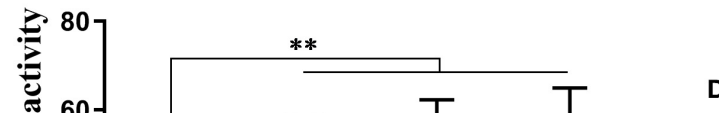

D

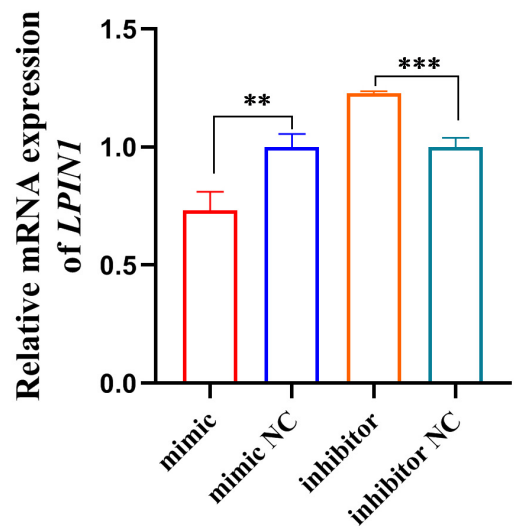

E
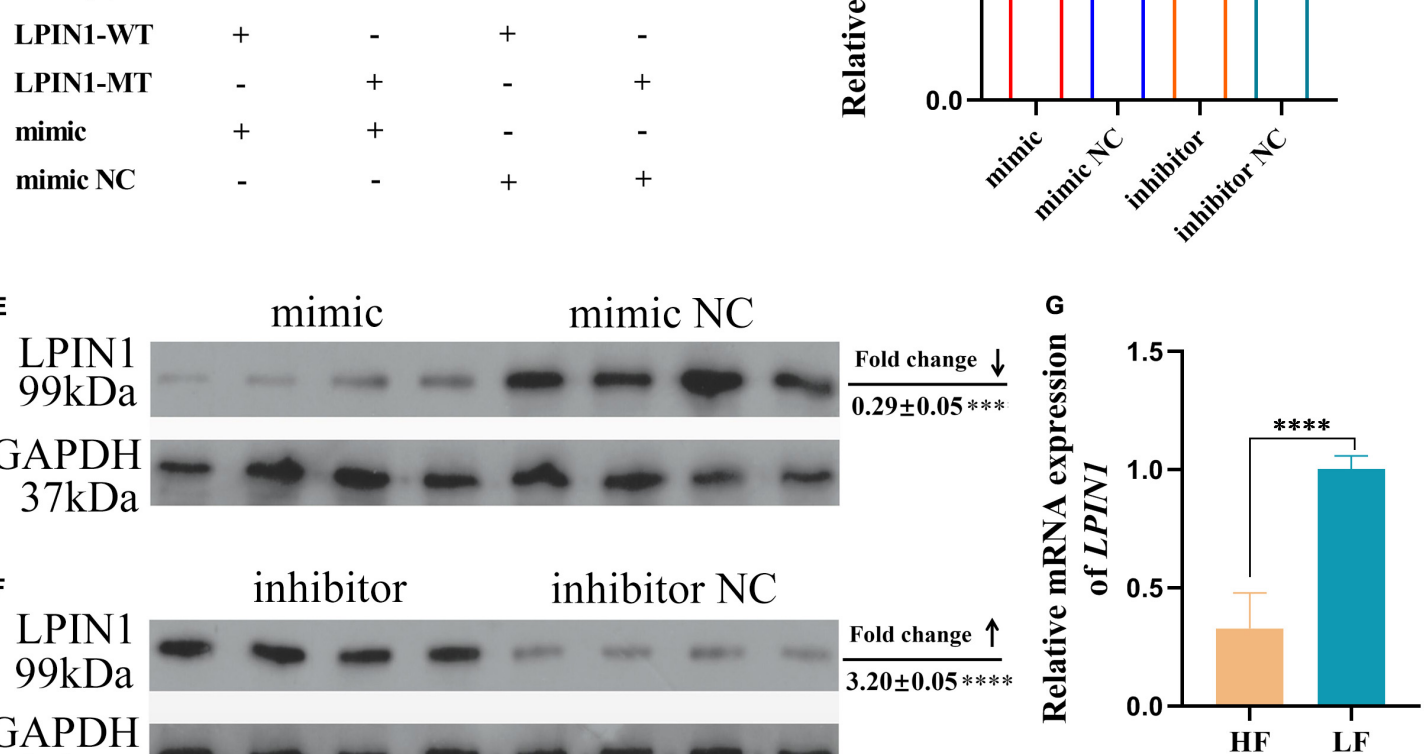
$37 \mathrm{kDa}$

FIGURE 6 | LPIN1 is a target gene of miR-429-3p. (A) The target relationship prediction between miR-429-3p and LPIN1 3'UTR. (B) The stability prediction between miR-429-3p and LPIN1 3'UTR. (C) Dual-luciferase reporter assay for miR-429-3p and LPIN1 3'UTR. (D) Relative mRNA expression of LPIN1 in ICP-1 cells treated with miR-429-3p mimic or inhibitor. (E,F) Relative protein level LPIN1 in ICP-1 cells treated with miR-429-3p mimic or inhibitor. (G) Relative mRNA expression of LPIN1 in high-fat chicken and low-fat chicken. Data presented as mean $\pm \mathrm{SEM},{ }^{\star} P<0.05$; ${ }^{\star \star} P<0.01 ;{ }^{* \star \star} P<0.001$; ${ }^{\star \star \star *} P<0.0001$; ns: no significance.

(Figures 8C,D). Results of the EdU assay showed that LPIN1 overexpression reduced the proportion of preadipocytes in the proliferation phase, whereas LPIN1 knockdown increased the same (Figures 8E,F). Therefore, LPIN1 exhibited an opposite effect to that of miR-429-3p and was speculated to inhibit preadipocyte proliferation.

\section{LPIN1 Inhibits Preadipocyte Differentiation by Suppressing PPAR $\gamma$ Pathway}

We also determined the differential expression of LPIN1 during adipocyte differentiation from preadipocytes. The expression of LPIN1 was significantly lower in differentiation-induced preadipocytes than before inducing differentiation (Figure 9A). Further, Oil red $\mathrm{O}$ staining showed the negative effect of LPIN1 on lipid droplet formation (Figures 9B-E). Based on the lower expression of LPIN1 in differentiation-induced preadipocytes and abdominal fat tissues of high-fat individuals, and the relationship between LPIN1 and miR-429-3p, we hypothesized that the low expression of LPIN1 is responsible for PPAR $\gamma$ pathway activation. Moreover, $\mathrm{qPCR}$ results indicated that the overexpression of LPIN1 suppressed the expression of $L P L, P P A R \gamma$, and $A D I P O$, three critical genes related to preadipocyte differentiation in $\operatorname{PPAR} \gamma$ pathway, along with the expression of $C / E B P \alpha$ and $C / E B P \beta$ (Figure 9F). On the 

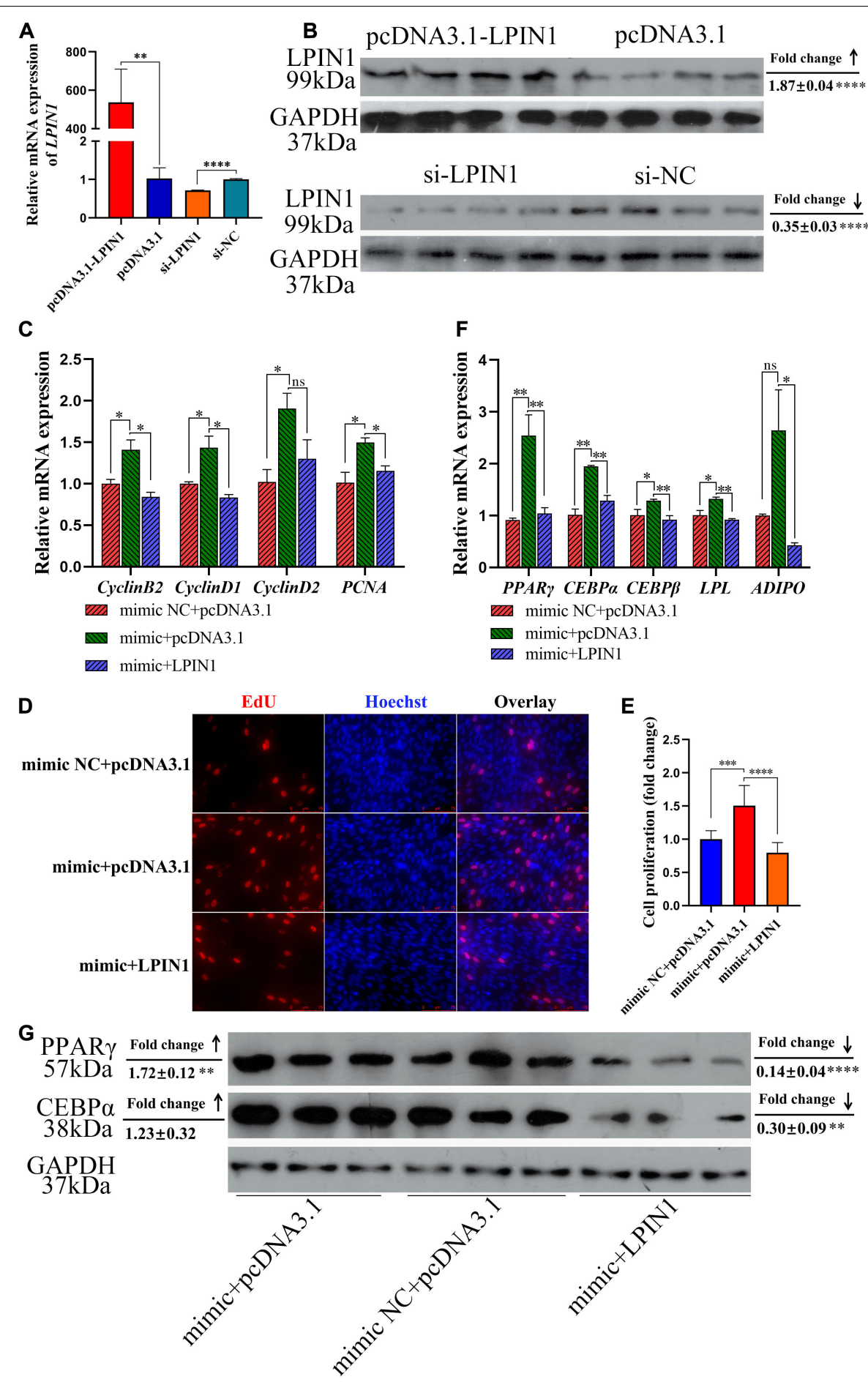

FIGURE 7 | LPIN1 plays a pivotal role on preadipocytes proliferation and differentiation regulated by miR-429-3p. (A) Transfection efficiency of pcDNA3.1-LPIN1 and si-LPIN1 on mRNA level. (B) Transfection efficiency of pcDNA3.1-LPIN1 and si-LPIN1 on protein level. (C) The mRNA expression of cell cycle-promoting genes in ICP-1 treated with mimic NC + pcDNA3.1, mimic + pcDNA3.1, and mimic + pcDNA3.1-LPIN1. (D) The mRNA expression of preadipocyte differentiation-related genes in ICP-1 treated with mimic NC + pcDNA3.1, mimic + pcDNA3.1, and mimic + pcDNA3.1-LPIN1. (E,F) EdU assay performed in ICP-1 treated with mimic NC + pcDNA3.1, mimic + pcDNA3.1, and mimic + pcDNA3.1-LPIN1. (G) The protein expression of PPARy and C/EBPa in ICP-1 treated with mimic NC + pcDNA3.1, mimic + pcDNA3.1 and mimic + pcDNA3.1-LPIN1. Data presented as mean \pm SEM, ${ }^{\star} P<0.05 ;{ }^{\star \star} P<0.01 ;{ }^{* \star \star} P<0.001 ;{ }^{* \star \star \star} P<0.0001 ;$ ns: no significance. 

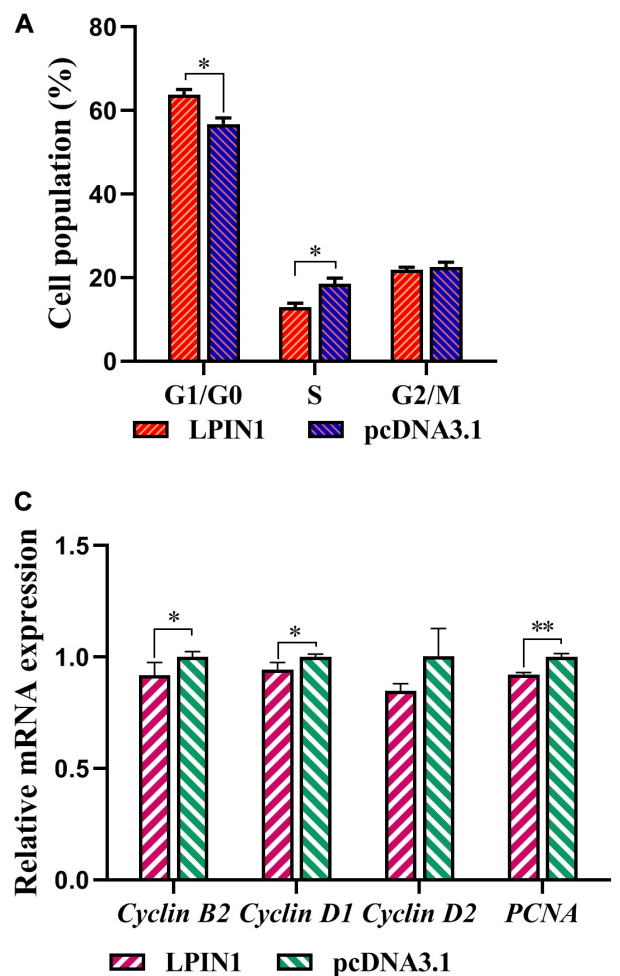

E

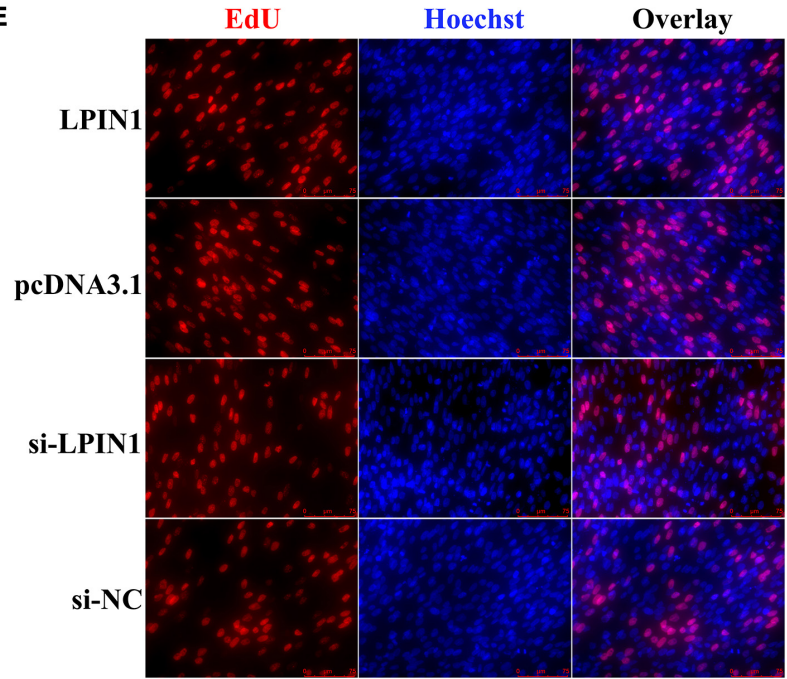

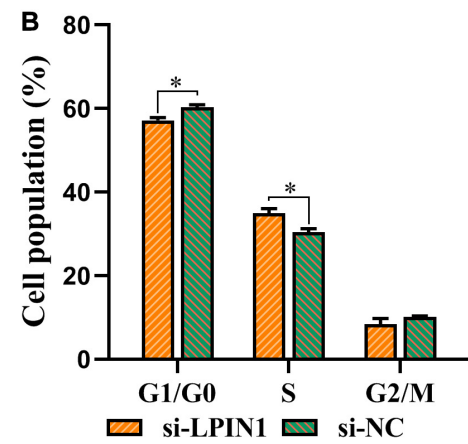

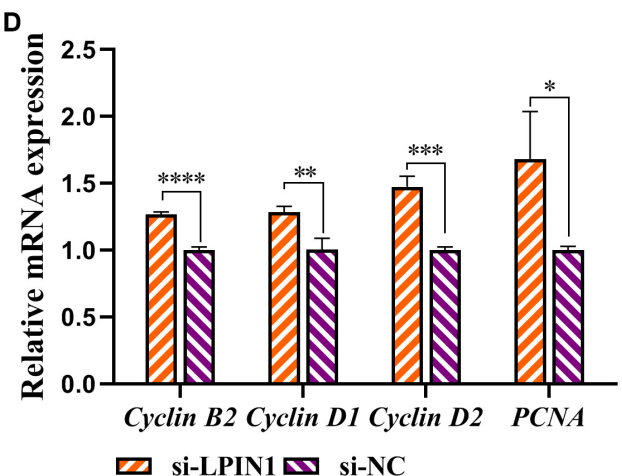

si-LPIN1 $\mathbf{\square}$ si-NC

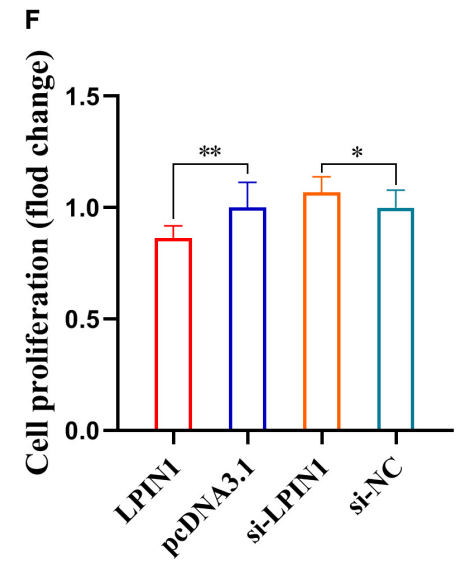

FIGURE 8 | LPIN1 suppresses preadipocytes proliferation. (A,B) Flow cytometry for cell cycle detection of ICP-1 cells. (C,D) Relative mRNA expression of Cyclin B2, Cyclin D1, Cyclin D2, and PCNA in ICP-1 cells. (E,F) EdU staining for cell proliferation of ICP-1 cells. Data presented as mean \pm SEM, ${ }^{\star} P<0.05$; ${ }^{\star \star} P<0.01$; ${ }^{\star \star \star} P<0.001 ;{ }^{* \star \star \star} P<0.0001$; ns: no significance.

contrary, the knockdown of LPIN1 upregulated these genes (Figure 9G). The protein levels of CEPB $\alpha$ and $\operatorname{PPAR} \gamma$ were reduced by overexpressing LPIN1, while knocking down LPIN1 increased their protein expression (Figure 9H). Further, as shown in Figures 8F,G, LPIN1 inhibited the formation of lipid droplets during preadipocyte differentiation. These effects of LPIN1 are opposite to those of miR-429-3p. Collectively, these results establish that the low expression of LPIN1 promotes the activation of PPAR $\gamma$ pathway and facilitates preadipocyte differentiation.

\section{DISCUSSION}

In chickens, abdominal fat is a major adipose tissue, and affects meat yield and quality. Recently, miRNAs have been shown 


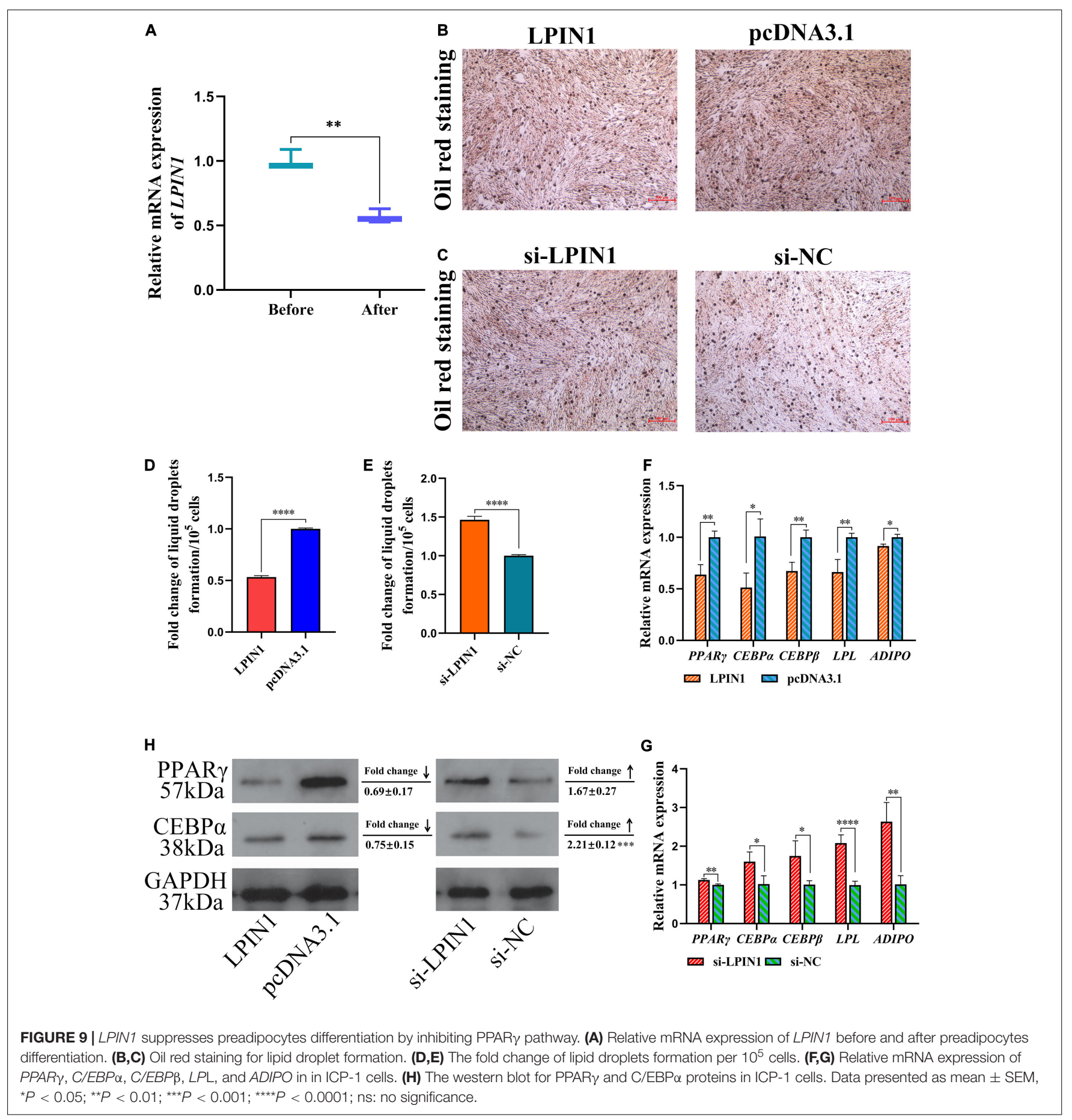

to be closely involved in various biological processes including adipogenesis (Alberti et al., 2018; Guay et al., 2019; LorenteCebrian et al., 2019). In this study, using sequencing techniques, we identified a miRNA (miR-429-3p) and its potential target gene (LPIN1), which show significant relation to the process of abdominal adipose deposition. Based on our sequencing data, we designed a series of experiments at molecular and cellular levels to explore the role and the interaction of miR-429-3p and LPIN1 in adipogenesis. Our results showed that miR-429-3p facilitated preadipocyte proliferation and differentiation. Additionally, miR429-3p was shown to target and suppress LPIN1, which exerted an opposite effect to that of miR-429-3p by inhibiting preadipocyte proliferation and differentiation. Notably, miR-429-3p/LPIN1 axis was functionally verified to modulate adipogenesis via PPAR $\gamma$ Pathway.

Our mRNA sequencing revealed multiple candidate genes related to lipid metabolism, including SLC27A2, ACSL6, ABHD5, LPIN1, ADORA1, HNF4A, and SQLE. In addition, we performed 
miRNA sequencing to screen some differentially expressed miRNAs which may be involved lipid metabolism, including miR-122b-3p, miR-126-5p, miR-122-5p, miR-148a-3p, miR$148 \mathrm{~b}-3 \mathrm{p}$, and miR-429-3p. DEMs-DEGs networks showed that miR-429-3p targeted LPIN1 in chicken abdominal fat tissue, which indicates that miR-429-3p/LPIN1 axis may regulate adipogenesis. miR-429-3p was significantly upregulated in high abdominal fat broilers and highly expressed after the differentiation from preadipocyte to adipocyte, indicating a potential regulatory effect on chicken adipose deposition. Given the evidence that miR-429-3p might be a significantly involved in the process of abdominal adipose deposition, we further investigated its role in this biological process. In the past decades, role of miR-429-3p has been extensively explored in tumorigenesis (Wu et al., 2018, 2019; Shen et al., 2019; Chen et al., 2020). However, little is known about its role in adipogenesis. A study by Tao et al. studied the function of miR-200b/a/429 in the regulation of metabolism in adipocytes (Tao et al., 2016). They generated adipocyte-specific miR200b/a/429 knockout mice and found that adipocyte miR200b/a/429 deficiency resulted in increased adiposity. Their finding suggests that miR-200b/a/429 could hinder high-fatdiet-induced obesity. However, our sequencing data showed that miR-429-3p was significantly upregulated in high-fat chickens. Flow cytometry suggested a promotion of miR429-3p on preadipocyte cell cycle transition from G1 phase to $\mathrm{S}$ phase. Abdominal fat rate is influenced by abdominal fat weight and body weight, both of which are important growth traits of chickens (Ren et al., 2019; Li et al., 2020). Nucleotide polymorphisms of cell cycle related gene have been reported to be associated with chicken growth traits (Li et al., 2019), the qPCR results in our study manifested miR-429-3p indeed increased cell cycle-promoting genes expression. Taking EdU results together, we proved that miR-429-3p promoted preadipocyte proliferation. The contradictory results between the study mentioned above and our study may be attributed to the differences in animal models and experimental methods used. The animal model used in the previous study was adipocytespecific miR-200b/a/429 knockout mice using a Cre-loxP system, in which Cre expression was driven by the aP2 promoter. In contrast, we used a chicken precursor adipocyte (ICP-1) as experiment cell model. Tao et al. analyzed the changes in body composition, metabolic parameters, energy homeostasis, glucose tolerance, and insulin sensitivity in miR-200b/a/429 knockout mice and wild-type mice after a high-fat diet, and concluded that miR-200b/a/429 knockout mice gained more body weight than the wild-type mice with increased adiposity, decreased glucose tolerance and insulin sensitivity. In another study, miR-429 has been shown to promote the proliferation of porcine preadipocyte (Peng et al., 2016), which proved by flow cytometry, qPCR and western blot of some cell cyclerelated genes and EdU assay. The results of Peng et al. are consistent with ours. miR-429 also played a pivotal role on browning of white adipose tissue (Ye et al., 2020), indicating its potential role on preadipocyte differentiation. In our present study, we utilized qPCR, western blotting and oil red $\mathrm{O}$ staining to determine the effect of miR-429-3p on differentiation of ICP-1 through gain or loss of function experiments. We revealed the promotion effect of miR-429-3p on PPAR $\gamma$ signaling pathway at protein and mRNA levels. Furthermore, miR-429-3p overexpression upregulated preadipocyte differentiation-related genes expression and contributed to lipid droplets formation.

We further investigated the role of miR-429-3p in adipogenesis by focusing on the reports of an earlier study which concluded that miRNAs exert biological functions by downregulating the expression of target genes (Hausser and Zavolan, 2014). Taking DEMs-DEGs network analysis together, we predicted the potential target gene of miR-429$3 \mathrm{p}$ in preadipocytes and determined that LPIN1 expression was targeted by miR-429-3p. To confirm our hypothesis, we investigated the effects of overexpression or knockdown of LPIN1 on preadipocyte proliferation and differentiation. The results demonstrated that LPIN1 exerted a completely opposite effect to that of miR-429-3p, and the mRNA and protein expression of LPIN1 was repressed by miR-429$3 p$. Dual-luciferase reporter assay provided the evidence of stable binding between miR-429-3p and LPIN1 3'UTR. A study by van Harmelen et al. (2007) showed that LPIN1 expression was reduced in obesity, was upregulated following weight reduction in obese subjects, and was downregulated in women with the metabolic syndrome. These results are in line with our data which demonstrated a lower expression of LPIN1 in high-fat chicken. To determine whether LPIN1 play the pivotal effect on preadipocyte proliferation and differentiation, we carried out the rescue experiments. And the result showed that the expression of LPIN1 reversed the promotion of miR-429-3p on preadipocyte proliferation. In addition, LPIN1 also fell back the PPAR $\gamma$ mRNA and protein levels, upregulated by miR-429-3p. In lipin-deficient and transgenic mouse models, lipin is identified as a key regulator in the adipocyte differentiation process in which PPAR $\gamma$ and $\mathrm{C} / \mathrm{EBP} \alpha$ are actively involved (Phan et al., 2004). In the chicken preadipocyte model, we also showed that LPIN1 protein is expressed during the preadipocyte differentiation process and is inversely related to PPAR $\gamma$ and $\mathrm{C} / \mathrm{EBP} \alpha$ expression, indicating that LPIN1 may have inhibitory effect on chicken adipogenesis. The effect of LPIN1 on abdominal fat deposition in chickens is completely opposite to that in mice, and the specific mechanism causing this difference is not clear, which needs further study in the future.

In conclusion, we show that miR-429-3p is upregulated, whereas LPIN1 is downregulated in high-fat chickens. Further, we showed the relationship between miR-429-3p and LPIN1 and established that miR-429-3p promoted preadipocyte proliferation and differentiation by inhibiting LPIN1 activity. The findings in this study reveal a novel miR-429-3p/LPIN1 axis involved in the progression of adipogenesis via the PPAR $\gamma$ pathway.

\section{DATA AVAILABILITY STATEMENT}

The datasets presented in this study can be found in online repositories. The names of the repository/repositories and 
accession number(s) can be found below: https://www.ncbi. nlm.nih.gov/, PRJNA657369 and https://www.ncbi.nlm.nih. gov/, PRJNA656618.

\section{ETHICS STATEMENT}

The animal study was reviewed and approved by the Agricultural University Animal Care Committee of South China Agricultural University.

\section{AUTHOR CONTRIBUTIONS}

$\mathrm{XC}$ and LG were responsible for research designing and for most of the experiments and manuscript writing. QW participated in the part of experiments and data analysis. WH reviewed and modified the manuscript. ML, KL, and JJ participated in the data analysis and part of experiments. SL analyzed the sequencing data. QN participated in the animal experiment. WL and XZ were responsible for sample collection. QL carried out the design of the whole research and guided the research progress. All authors contributed to the article and approved the submitted version.

\section{REFERENCES}

Alberti, C., Manzenreither, R. A., Sowemimo, I., Burkard, T. R., Wang, J., Mahofsky, K., et al. (2018). Cell-type specific sequencing of microRNAs from complex animal tissues. Nat. Methods 15, 283-289. doi: 10.1038/nmeth.4610

Asghari, G., Mirmiran, P., Yuzbashian, E., and Azizi, F. (2017). A systematic review of diet quality indices in relation to obesity. Br. J. Nutr. 117, 1055-1065. doi: 10.1017/S0007114517000915

Barnett, T. A., Kelly, A. S., Young, D. R., Perry, C. K., Pratt, C. A., Edwards, N. M., et al. (2018). Sedentary behaviors in today's youth: approaches to the prevention and management of childhood obesity: a scientific statement from the american heart association. Circulation 138, e142-e159. doi: 10.1161/CIR. 0000000000000591

Bartel, D. P. (2004). MicroRNAs: genomics, biogenesis, mechanism, and function. Cell 116, 281-297. doi: 10.1016/s0092-8674(04)00045-5

Bray, G. A. (2004). Medical consequences of obesity. J. Clin. Endocrinol. Metab. 89, 2583-2589. doi: 10.1210/jc.2004-0535

Brun, R. P., Tontonoz, P., Forman, B. M., Ellis, R., Chen, J., Evans, R. M., et al. (1996). Differential activation of adipogenesis by multiple PPAR isoforms. Genes Dev. 10, 974-984. doi: 10.1101/gad.10.8.974

Bugianesi, E. (2020). Fatty liver disease: putting the spotlight on a silent menace for young adults. Lancet Gastroenterol. Hepatol. 5, 236-238. doi: 10.1016/S24681253(19)30420-0

Campeau, P. M., Astapova, O., Martins, R., Bergeron, J., Couture, P., Hegele, R. A., et al. (2012). Clinical and molecular characterization of a severe form of partial lipodystrophy expanding the phenotype of PPARgamma deficiency. J. Lipid Res. 53, 1968-1978. doi: 10.1194/jlr.P025437

Chen, Z., Chen, J., Wa, Q., He, M., Wang, X., Zhou, J., et al. (2020). Knockdown of circ_0084043 suppresses the development of human melanoma cells through miR-429/tribbles homolog 2 axis and Wnt/beta-catenin pathway. Life Sci. 243:117323. doi: 10.1016/j.lfs.2020.117323

Fedorenko, A., Lishko, P. V., and Kirichok, Y. (2012). Mechanism of fatty-aciddependent UCP1 uncoupling in brown fat mitochondria. Cell 151, 400-413. doi: 10.1016/j.cell.2012.09.010

Fouad, A. M., and El-Senousey, H. K. (2014). Nutritional factors affecting abdominal fat deposition in poultry: a review. Asian Austral. J. Anim. Sci. 27, 1057-1068. doi: 10.5713/ajas.2013.13702

\section{FUNDING}

This research was funded by the Guangdong Basic and Applied Basic Research Fund Project (2019B1515210005) and Guangdong Province Modern Agricultural Industry Technology System Project (2019KJ128).

\section{SUPPLEMENTARY MATERIAL}

The Supplementary Material for this article can be found online at: https://www.frontiersin.org/articles/10.3389/fcell.2020. 595637/full\#supplementary-material

Supplementary Figure 1 | The first nucleotide bias of small RNA.

Supplementary Table 1 | Primers sequence information.

Supplementary Table 2 | Data quality statistics and mapping efficiency.

Supplementary Table $\mathbf{3}$ | The enriched pathways related to fat deposition.

Supplementary Table 4 | The top-enrich pathways for DEGs in

DAVID 6.8 platform.

Supplementary Table $\mathbf{5}$ | The lipid metabolism-related pathways enriched in IPA platform.

Supplementary Table 6 | miRNA count in small RNA-seq.

Goodarzi, M. O. (2018). Genetics of obesity: what genetic association studies have taught us about the biology of obesity and its complications. Lancet Diabetes Endocrinol. 6, 223-236. doi: 10.1016/S2213-8587(17)30200-0

Guay, C., Kruit, J. K., Rome, S., Menoud, V., Mulder, N. L., Jurdzinski, A., et al. (2019). Lymphocyte-derived exosomal MicroRNAs promote pancreatic beta cell death and may contribute to type 1 diabetes development. Cell Metab. 29, 348-361. doi: 10.1016/j.cmet.2018.09.011

Hasuwa, H., Ueda, J., Ikawa, M., and Okabe, M. (2013). miR-200b and miR-429 function in mouse ovulation and are essential for female fertility. Science 341, 71-73. doi: 10.1126/science.1237999

Hausser, J., and Zavolan, M. (2014). Identification and consequences of miRNAtarget interactions-beyond repression of gene expression. Nat. Rev. Genet. 15, 599-612. doi: 10.1038/nrg3765

Heianza, Y., and Qi, L. (2017). Gene-diet interaction and precision nutrition in obesity. Int. J. Mol. Sci. 18:787. doi: 10.3390/ijms18040787

Homan, E. A., Kim, Y. G., Cardia, J. P., and Saghatelian, A. (2011). Monoalkylglycerol ether lipids promote adipogenesis. J. Am. Chem. Soc. 133, 5178-5181. doi: 10.1021/ja111173c

Hruby, A., and Hu, F. B. (2015). The epidemiology of obesity: a big picture. Pharmacoeconomics 33, 673-689. doi: 10.1007/s40273-014-0243-x

Jaacks, L. M., Vandevijvere, S., Pan, A., McGowan, C. J., Wallace, C., Imamura, F., et al. (2019). The obesity transition: stages of the global epidemic. Lancet Diabetes Endocrinol. 7, 231-240. doi: 10.1016/S2213-8587(19)30026-9

James, W. (2018). Obesity: a global public health challenge. Clin. Chem. 64, 24-29. doi: 10.1373/clinchem.2017.273052

Krol, J., Loedige, I., and Filipowicz, W. (2010). The widespread regulation of microRNA biogenesis, function and decay. Nat. Rev. Genet. 11, 597-610. doi: $10.1038 / \operatorname{nrg} 2843$

Kuhnen, P., Krude, H., and Biebermann, H. (2019). Melanocortin-4 receptor signalling: importance for weight regulation and obesity treatment. Trends Mol. Med. 25, 136-148. doi: 10.1016/j.molmed.2018.12.002

Lauby-Secretan, B., Scoccianti, C., Loomis, D., Grosse, Y., Bianchini, F., and Straif, K. (2016). Body fatness and cancer-viewpoint of the IARC working group. N. Engl. J. Med. 375, 794-798. doi: 10.1056/NEJMsr1606602

Lavie, C. J., Ozemek, C., Carbone, S., Katzmarzyk, P. T., and Blair, S. N. (2019). Sedentary behavior, exercise, and cardiovascular health. Circ. Res. 124, 799-815. doi: 10.1161/CIRCRESAHA.118.312669 
Lefterova, M. I., Zhang, Y., Steger, D. J., Schupp, M., Schug, J., Cristancho, A., et al. (2008). PPARgamma and C/EBP factors orchestrate adipocyte biology via adjacent binding on a genome-wide scale. Genes Dev. 22, 2941-2952. doi: 10.1101/gad.1709008

Li, W., Jing, Z., Cheng, Y., Wang, X., Li, D., Han, R., et al. (2020). Analysis of four complete linkage sequence variants within a novel lncRNA located in a growth QTL on chromosome 1 related to growth traits in chickens. J. Anim. Sci. 98:skaa122. doi: 10.1093/jas/skaa122

Li, W., Liu, D., Tang, S., Li, D., Han, R., Tian, Y., et al. (2019). A multiallelic indel in the promoter region of the Cyclin-dependent kinase inhibitor 3 gene is significantly associated with body weight and carcass traits in chickens. Poult. Sci. 98, 556-565. doi: 10.3382/ps/pey404

Li, Z., Lan, X., Guo, W., Sun, J., Huang, Y., Wang, J., et al. (2012). Comparative transcriptome profiling of dairy goat microRNAs from dry period and peak lactation mammary gland tissues. PLoS One 7:e52388. doi: 10.1371/journal. pone. 0052388

Liu, D., Archer, N., Duesing, K., Hannan, G., and Keast, R. (2016). Mechanism of fat taste perception: association with diet and obesity. Prog. Lipid Res. 63, 41-49. doi: 10.1016/j.plipres.2016.03.002

Lorente-Cebrian, S., Gonzalez-Muniesa, P., Milagro, F. I., and Martinez, J. A. (2019). MicroRNAs and other non-coding RNAs in adipose tissue and obesity: emerging roles as biomarkers and therapeutic targets. Clin. Sci. 133, 23-40. doi: 10.1042/CS20180890

Madsen, M. S., Siersbaek, R., Boergesen, M., Nielsen, R., and Mandrup, S. (2014). Peroxisome proliferator-activated receptor gamma and C/EBPalpha synergistically activate key metabolic adipocyte genes by assisted loading. Mol. Cell. Biol. 34, 939-954. doi: 10.1128/MCB.01344-13

Mohr, A. M., and Mott, J. L. (2015). Overview of microRNA biology. Semin. Liver Dis. 35, 3-11. doi: 10.1055/s-0034-1397344

Peng, Y., Chen, F. F., Ge, J., Zhu, J. Y., Shi, X. E., Li, X., et al. (2016). miR-429 inhibits differentiation and promotes proliferation in porcine preadipocytes. Int. J. Mol. Sci. 17:2047. doi: 10.3390/ijms17122047

Phan, J., Peterfy, M., and Reue, K. (2004). Lipin expression preceding peroxisome proliferator-activated receptor-gamma is critical for adipogenesis in vivo and in vitro. J. Biol. Chem. 279, 29558-29564. doi: 10.1074/jbc.M403506200

Qasim, A., Turcotte, M., de Souza, R. J., Samaan, M. C., Champredon, D., Dushoff, J., et al. (2018). On the origin of obesity: identifying the biological, environmental and cultural drivers of genetic risk among human populations. Obes. Rev. 19, 121-149. doi: 10.1111/obr.12625

Qiu, J., Zhang, C., Borgquist, A., Nestor, C. C., Smith, A. W., Bosch, M. A., et al. (2014). Insulin excites anorexigenic proopiomelanocortin neurons via activation of canonical transient receptor potential channels. Cell. Metab. 19, 682-693. doi: 10.1016/j.cmet.2014.03.004

Ren, T., Li, W., Liu, D., Liang, K., Wang, X., Li, H., et al. (2019). Two insertion/deletion variants in the promoter region of the QPCTL gene are significantly associated with body weight and carcass traits in chickens. Anim. Genet. 50, 279-282. doi: 10.1111/age.12741
Saxton, S. N., Clark, B. J., Withers, S. B., Eringa, E. C., and Heagerty, A. M. (2019). Mechanistic links between obesity, diabetes, and blood pressure: role of perivascular adipose tissue. Physiol. Rev. 99, 1701-1763. doi: 10.1152/physrev. 00034.2018

Shen, J., Hong, L., Yu, D., Cao, T., Zhou, Z., and He, S. (2019). LncRNA XIST promotes pancreatic cancer migration, invasion and EMT by sponging miR429 to modulate ZEB1 expression. Int. J. Biochem. Cell. Biol. 113, 17-26. doi: 10.1016/j.biocel.2019.05.021

Subramaniam, S., Jeet, V., Clements, J. A., Gunter, J. H., and Batra, J. (2019). Emergence of MicroRNAs as key players in cancer cell metabolism. Clin. Chem. 65, 1090-1101. doi: 10.1373/clinchem.2018.299651

Swinburn, B. A., Sacks, G., Hall, K. D., McPherson, K., Finegood, D. T., Moodie, M. L., et al. (2011). The global obesity pandemic: shaped by global drivers and local environments. Lancet 378, 804-814. doi: 10.1016/S0140-6736(11)60813-1

Tao, C., Ren, H., Xu, P., Cheng, J., Huang, S., Zhou, R., et al. (2016). Adipocyte miR$200 \mathrm{~b} / \mathrm{a} / 429$ ablation in mice leads to high-fat-diet-induced obesity. Oncotarget 7, 67796-67807. doi: 10.18632/oncotarget.12080

Tontonoz, P., Hu, E., Graves, R. A., Budavari, A. I., and Spiegelman, B. M. (1994). mPPAR gamma 2: tissue-specific regulator of an adipocyte enhancer. Genes Dev. 8, 1224-1234. doi: 10.1101/gad.8.10.1224

van Harmelen, V., Ryden, M., Sjolin, E., and Hoffstedt, J. (2007). A role of lipin in human obesity and insulin resistance: relation to adipocyte glucose transport and GLUT4 expression. J. Lipid Res. 48, 201-206. doi: 10.1194/jlr.M600272JLR200

Wu, C. L., Ho, J. Y., Hung, S. H., and Yu, D. S. (2018). miR-429 expression in bladder cancer and its correlation with tumor behavior and clinical outcome. Kaohsiung J. Med. Sci. 34, 335-340. doi: 10.1016/j.kjms.2018.01.001

Wu, G., Zheng, H., Xu, J., Guo, Y., Zheng, G., Ma, C., et al. (2019). miR-429 suppresses cell growth and induces apoptosis of human thyroid cancer cell by targeting ZEB1. Artif. Cells Nanomed Biotechnol. 47, 548-554. doi: 10.1080/ 21691401.2018.1564320

Ye, C., Duan, J., Zhang, X., Yao, L., Song, Y., Wang, G., et al. (2020). Cold-induced Yes-associated-protein expression through miR-429 mediates the browning of white adipose tissue. Sci. China Life Sci. doi: 10.1007/s11427-020-1779-2 [Epub ahead of print].

Conflict of Interest: The authors declare that the research was conducted in the absence of any commercial or financial relationships that could be construed as a potential conflict of interest.

Copyright (c) 2020 Chao, Guo, Wang, Huang, Liu, Luan, Jiang, Lin, Nie, Luo, Zhang and Luo. This is an open-access article distributed under the terms of the Creative Commons Attribution License (CC BY). The use, distribution or reproduction in other forums is permitted, provided the original author(s) and the copyright owner(s) are credited and that the original publication in this journal is cited, in accordance with accepted academic practice. No use, distribution or reproduction is permitted which does not comply with these terms. 